\title{
Altered Mediator dynamics during heat shock in budding yeast
}

Debasish Sarkar ${ }^{1}$, Z. Iris Zhu ${ }^{3}$, Emily Paul1\#, David Landsman ${ }^{3}$, and Randall H. Morse ${ }^{1,2^{*}}$

${ }^{1}$ Wadsworth Center, New York State Department of Health, Albany, New York 12201-0509

${ }^{2}$ Department of Biomedical Sciences, University at Albany School of Public Health, Albany,

New York, 12201-0509

${ }^{3}$ Computational Biology Branch, National Center for Biotechnology Information, National

Library of Medicine, NIH, Bethesda, MD 20814

\#Current address: Sophia Genetics, 185 Dartmouth St., Boston, MA 02116

*Corresponding author: randall.morse@health.ny.gov

Running title: Mediator dynamics during heat shock in yeast

Keywords: Mediator, transcription, stress response, $\mathrm{CdCl}_{2}$, RNA polymerase II, ChIP-seq, Kin28 


\begin{abstract}
The Mediator complex is central to transcription by RNA polymerase II (Pol II) in eukaryotes. In yeast, Mediator is recruited by activators via its tail module and then facilitates assembly of the pre-initiation complex (PIC), including Pol II, setting the stage for productive transcription. Mediator occupies proximal promoter regions only transiently prior to Pol II escape; interruption of the transcription cycle by inactivation or depletion of Kin28 inhibits Pol II escape and stabilizes Mediator occupancy at promoters. However, whether Mediator occupancy and dynamics differ for gene cohorts induced by stress or alternative growth conditions has not been examined on a genome-wide scale. Here we investigate Mediator occupancy following heat shock or $\mathrm{CdCl}_{2}$ induction, with or without depletion of Kin28. We find that Pol II occupancy exhibits similar dependence on Mediator under normal and heat shock conditions; however, Mediator occupancy does not increase upon Kin28 depletion at most genes active during heat shock, indicating altered dynamics. Furthermore, Mediator occupancy persists at genes repressed by heat shock or $\mathrm{CdCl}_{2}$ induction and exhibits peaks upstream of the proximal promoter whether or not Kin28 is depleted, suggesting that Mediator is recruited by activators but is unable to engage PIC components at these repressed targets. Finally, we show a reduced dependence on PIC components for Mediator occupancy at promoters after heat shock, further supporting an altered dynamics or stronger engagement with activators under these conditions.
\end{abstract}




\section{Introduction}

Transcription of protein-coding genes in eukaryotes is a complex process involving recruitment of coactivators, assembly of the pre-initiation complex (PIC), including RNA polymerase II (Pol II), and transition from promoter melting and transcription initiation to productive transcriptional elongation (Schier and Taatjes 2020). The Mediator complex, a large, multi-subunit complex present in plants, animals, and single-celled eukaryotes, is central to this process (Jeronimo and Robert 2017; Soutourina 2018). In yeast, Mediator has been divided into head, middle, tail, and cyclin-CDK modules on the basis of structural and genetic data (Plaschka et al. 2016). Although recent, high-resolution cryo-EM investigations have revised and added nuance to Mediator structure (Tsai et al. 2014; Wang et al. 2014; Plaschka et al. 2015), it remains the case that functional attributes of yeast Mediator segregate, to some extent, to distinct modules (van de Peppel et al. 2005; Knoll et al. 2018).

Functionally, activators recruit yeast Mediator to upstream activation sites (UAS regions) via the tail module (in particular the tail module triad of Med2-Med3-Med15); subunits in the middle and head module then engage PIC components, including Pol II, to facilitate PIC assembly (Jeronimo and Robert 2017; Knoll et al. 2018; Soutourina 2018). This process entails bridging between the UAS and the proximal promoter, where the PIC is assembled, by a single Mediator complex (Jeronimo et al. 2016; Petrenko et al. 2016). Mediator ChIP signal at UAS regions is variable in strength and not well correlated with transcription levels, while under normal conditions, Mediator association with proximal promoter regions is transient and difficult to detect by ChIP (Fan et al. 2006; Fan and Struhl 2009; Jeronimo and Robert 2014; Wong et al. 2014; Paul et al. 2015b). For example, genes 
encoding ribosomal proteins (RP genes) depend on Mediator for Pol II recruitment under normal growth conditions, yet many exhibit weak Mediator ChIP signal in spite of being transcriptionally highly active. The conclusion that Mediator does occupy proximal promoters, if normally only transiently, is based on experiments showing that depletion or inactivation of Kin28, which inhibits promoter escape by Pol II, results in robust Mediator ChIP signal at gene promoters (Jeronimo and Robert 2014; Wong et al. 2014). Consistent with this interpretation, Mediator occupancy at promoters under conditions of Kin28 depletion is reduced by simultaneous depletion of Pol II, Taf1, or TBP; in the latter case, increased Mediator ChIP signal is observed at UAS sites, indicating that TBP is required for transit of Mediator to promoters from its initial sites of recruitment (Knoll et al. 2018).

Mediator occupancy measured by ChIP during the normal transcription cycle (i.e. with Kin28 active) is much higher at induced genes that are controlled by strong activators, such as those activated by heat shock or growth in galactose (Fan et al. 2006; Fan and Struhl 2009; Kim and Gross 2013). This could reflect stronger interactions between Mediator and activators, and hence less dependence on PIC components or other factors for stabilizing Mediator occupancy, at strongly induced genes than at constitutively active genes during growth in rich medium (YPD). Increased Mediator occupancy has been observed at a few individual promoters induced by heat shock following Kin28 depletion (Petrenko et al. 2016); however, no genome-wide analysis has been reported examining whether promoter occupancy by Mediator is stabilized by Kin28 depletion at strongly induced genes or depends on PIC components as it does in yeast grown in rich medium (YPD). In this work, we examine genome-wide Mediator occupancy following two 
perturbations, heat shock and $\mathrm{CdCl}_{2}$ administration, that induce large and rapid genomewide transcriptional responses.

\section{Results}

\section{Pol II recruitment upon heat shock depends on Mediator}

To identify genes most active after heat shock and most highly induced by heat shock, we performed ChIP-seq to determine Pol II occupancy in the commonly used laboratory strain BY4741, and in YFR1321, the parent strain to that used for anchor-away of Kin28, before and after 15 and 30 min of heat shock. Heat shock resulted in increased Pol II occupancy at targets of Hsf1, the primary transcription factor responding to heat shock, and the stress responsive activators Msn2 and Msn4, while Pol II occupancy was nearly completely lost from RP genes, consistent with previous determinations of mRNA levels and nascent RNA production following heat shock (Figure 1A) (Warner 1999; Gasch et al. 2000; Causton et al. 2001; Pincus et al. 2018). We observed a bias for Pol II occupancy towards the $5^{\prime}$ ends of genes after heat shock, as has been noted previously (Kim et al. 2010; McKinlay et al. 2011). K-means clustering revealed two groups of genes strongly upregulated by heat shock; these groups were both enriched for association with Hsf1 and Msn2 and Msn4 (Figure 1B). Transcription induced by Hsf1 has been reported to occur independently of Mediator (Lee and Lis 1998; McNeil et al. 1998), but we found a similar 23 fold reduction in Pol II occupancy at Hsf1 and Msn2/4 targets after heat shock upon depletion of the Mediator head module subunit Med17, using the anchor away method (see next section), as at other genes in the absence of heat shock, in agreement with recent results from the Struhl lab (Petrenko et al. 2017) (Figure 1C). 
Effect of Kin28 depletion on Mediator recruitment with and without heat shock

To examine Mediator dynamics at genes induced upon heat shock, we performed ChIP-seq against Med15, from the Mediator tail module triad, in yeast engineered to allow depletion of Kin28 from the cell nucleus using the anchor away method (kin28-AA yeast), and in the parent strain, YFR1321 (Jeronimo and Robert 2014; Wong et al. 2014; Knoll et al. 2018). (We used epitope-tagged derivatives of these strains (see Methods) to allow ChIP of Med15 and Med18; for simplicity, we refer to these strains by the parent strain names throughout the text and figure legends.) These strains harbor a tor1-1 mutation that abrogates the stress response normally accompanying exposure to rapamycin (Haruki et al. 2008). The kin28-AA yeast strain expresses Kin28 with a C-terminal FRB tag and the ribosomal subunit RPL13A C-terminally tagged with the FKBP12 fragment; upon administration of rapamycin, Kin28-FRB is tightly coupled to RPL13A-FKBP12 and is evicted from the nucleus following nuclear processing of RPL13A. The parent strain is identical except for lacking the FRB tag. Yeast cultures were grown to early log phase $\left(\mathrm{A}_{600}\right.$ 0.6-0.8), treated with rapamycin for $1 \mathrm{hr}$ to allow Kin28 depletion, and then subjected to heat shock by a rapid temperature shift for 15-30 minutes prior to cross-linking for ChIPseq analysis. Previous work has shown that $1 \mathrm{hr}$ of rapamycin treatment results in efficient depletion of target protein and of Kin28 in particular, and robust recruitment of Mediator has been observed after 5-30 minutes of heat shock (Haruki et al. 2008; Kim and Gross 2013; Wong et al. 2014; Knoll et al. 2018).

In the absence of heat shock, depletion of Kin28 resulted in an increase in Med15 ChIP signal at promoters of Hsf1 targets and RP genes, consistent with prior work 
(compare "Parent no HS" and "kin28-AAR no HS" in Figure 2A; see also Figure S1A for biological replicate experiment) (Jeronimo and Robert 2014; Wong et al. 2014). Many Hsf1 target genes are active even in the absence of heat shock (Gross et al. 1990; Solis et al. 2016; de Jonge et al. 2017; Pincus et al. 2018), so the association of Mediator with promoters of Hsf1 targets in the absence of heat shock, when Kin28 is depleted, is not unexpected. Heat shock resulted in an increased Med15 ChIP signal at Hsf1 and Msn2/4 targets in both the parent strain and the kin28-AA strain (Figure 2A). Quantitation of Med15 ChiP signal at RP genes and at the $\sim 300$ non-RP gene promoters having highest Med15 occupancy in non-heat-shocked cells after Kin28 depletion revealed an increase in signal in kin28AA compared to WT yeast, as expected; this increase was especially pronounced for RP genes (Figure 2B and Figure S1B). In contrast, increased Med15 occupancy was not observed upon Kin28 depletion in heat-shocked cells, either at Hsf1 targets or the 300 genes most highly occupied by Med15 in kin28AA yeast after heat shock (Figure 2A-B and Figure S1A-B). Examination of the 300 non-RP gene promoters with highest Pol II occupancy (in non-heat shocked and heat-shocked YFR1321 yeast) yielded similar results (Figure S1C), as did comparison of genes having similar Pol II occupancy in the absence and presence of heat shock (Figure S1D).

Consistent with the trends evident from Figures 2A-B, stronger induction of Med15 occupancy upon Kin28 depletion under non-heat-shock than under heat-shock conditions was observed upon inspection of browser scans at many genes (Figure 2C; Figure S2, YDJ1). At the same time, some promoters behave counter to this trend, reflecting the range of altered Med15 occupancy observed upon Kin28 depletion (Figure 2B; Figure S2, TPS1). Variable effects on Med15 occupancy increase upon Kin28 depletion were observed for 
Hsf1 and Msn2/4 targets as well as for genes induced by heat shock that are not known to be targets of either of these stress-responsive activators (Figure S2; Table S1). Genespecific increase in Mediator ChIP signal upon Kin28 depletion or inactivation is similarly evident in previous reports, but the cause of this remains unexplained (Jeronimo and Robert 2014; Knoll et al. 2018).

Under normal growth conditions in rich medium, many active gene promoters, most conspicuously those of many RP genes, exhibit little or no association with Mediator as measured by ChIP (Jeronimo and Robert 2014; Wong et al. 2014; Paul et al. 2015b). Some active genes, however, do exhibit Mediator ChIP signal at UAS regions under normal growth conditions; depletion or inactivation of Kin28 results in a shift in Mediator occupancy at these genes from UAS to proximal promoter (Jeronimo and Robert 2014; Wong et al. 2014). Correspondingly, we observed a shift towards the proximal promoter with little change in intensity of the Med15 ChIP signal upon Kin28 depletion in the absence of heat shock at a set of 498 genes, which we refer to as "UAS genes", identified as exhibiting Mediator occupancy of UAS regions under normal growth conditions (Figure 2D) (Jeronimo et al. 2016). In contrast, RP genes show greatly increased Mediator occupancy upon Kin28 depletion, along with a shift in the peak closer to the TSS (Figure 2D).

Taken together, these results indicate that Hsf1 targets that are active under nonheat-shocked conditions behave similarly to the large cohort of genes, exemplified by RP genes, that exhibit little or no Mediator signal unless Kin28 is depleted, while under heat shock conditions, Hsf1 and Msn2/4 targets behave more like the "UAS genes" at which Mediator ChIP signal is seen under normal growth conditions and exhibits modest increase upon depletion of Kin28. 
Mediator remains associated with promoters of genes repressed by heat shock

We were surprised to note a prominent Med15 peak upstream of RP genes following heat shock (Figure 2A), despite the near absence of Pol II occupancy (Figure 1A). This peak was unaffected in position by Kin28 depletion in heat shocked cells, and was shifted slightly upstream relative to the Med15 peak observed following Kin28 depletion in the absence of heat shock (Figure 2A and Figure 3A). Thus, upon heat shock, Mediator association with RP genes, under conditions of depleted Kin28, shifts from a proximal promoter location to an upstream position that closely coincides with that of the RP gene activator Rap1 (Fig. 3A and see below). A similar shift upstream in kin28AA yeast following heat shock was seen at RP genes for the Mediator head module Med18, also known as Srb5, with the signal also showing a somewhat greater decrease in intensity compared to that for Med15 (Figure 3B; Figure S3).

As mentioned earlier, the kin28AA strain and its parent, YFR1321, harbor the tor1-1 mutation, which prevents the normal stress response accompanying rapamycin administration (Haruki et al. 2008). Because the Tor pathway is intimately connected with ribosome function (Mayer and Grummt 2006), we considered the possibility that the unexpected association of Mediator with RP genes repressed by heat shock was a consequence of the tor1-1 mutation. We therefore performed ChIP-seq against Med15 and Med18 in the wild type strain BY4741 before and after 15 min of heat shock. Again, we observed peaks at RP genes for both Med15 and Med18 following heat shock (Figure 3C; Figure S3). Furthermore, as with the anchor-away parent strain YFR1321, Mediator ChIPseq signal at RP genes was stronger after heat shock. The peaks for Med15 and Med18 were 
nearly coincident with the peak observed for Rap1, which binds upstream of 127 out of 137 RP genes and is essential for recruitment of the pre-initiation complex to RP genes (Figure 3C) (Lieb et al. 2001; Mencia et al. 2002; Ansari et al. 2009; Zeevi et al. 2011; Knight et al. 2014; Reja et al. 2015).

The ChIP-seq peaks for Mediator subunits observed upstream of RP genes in both wild type and kin28-AA yeast after heat shock suggest that Mediator is recruited to these genes in heat shocked cells but does not transit to proximal promoters as it normally does in non-heat-shocked cells. Many non-RP genes are also repressed by heat shock (Figure 1) (Gasch et al. 2000; Causton et al. 2001). To assess whether Mediator occupies promoters of such genes after heat shock, we examined ChIP-seq signal at UAS genes-that is, the 498 genes found to exhibit discernible Mediator occupancy in wild type yeast (Jeronimo et al. 2016). We divided these genes into those showing decreased Pol II occupancy by at least 2fold (116 "UAS down" genes after removing RP genes) and the 231 "UAS not down" genes having a ratio of Pol II occupancy in heat shocked compared to non-heat-shocked cells $\geq 1$, and compared the Med15 signal at these two sets of genes before and after heat shock (Figure 4A). We observed Med15 signal for both the "UAS down" and "UAS not down" cohorts in YFR1321 yeast (parent strain for kin28AA) grown at $30^{\circ} \mathrm{C}$; upon heat shock, Med15 signal was unchanged for "UAS down" genes and showed a slight increase for "UAS not down" genes (Fig. 4A). Thus, these two cohorts behaved similarly with regard to Mediator occupancy in spite of their divergent behavior with regard to Pol II occupancy (Figure 4A).

We then examined association of Med15 and Med18 at these same cohorts under conditions of Kin28 depletion. Similar to our observations at RP genes, both subunits 
exhibited continued association with genes repressed by heat shock (Figure 4B, left panels, and Figure 4C). When cells were not heat shocked, Med18, from the head module, exhibited a narrow peak close to the TSS, while Med15, from the tail module, showed a broader peak extending from near the TSS to farther upstream. This is consistent with previous observations, and concordant with Mediator being recruited to upstream activating sites via the tail module and then transiting to the proximal promoter upon engagement of the head module with PIC components (Jeronimo et al. 2016; Petrenko et al. 2016; Knoll et al. 2018). Thus, because the tail module is in closer contact with the UAS and the head module in closer contact with the proximal promoter, cross-linking is more efficient for Med15 at the UAS and for Med18 near the TSS. Upon heat shock, both subunits exhibited peaks that were shifted upstream relative to the peaks seen in the absence of heat shock.

Altogether, these results indicate that Mediator remains associated with UAS regions of many genes that are repressed by heat shock, including RP genes, but is unable to recruit Pol II or to facilitate PIC formation (Vinayachandran et al. 2018).

Role of PIC components in Mediator association following heat shock

We next addressed the role of PIC components TBP, TFIID, and Pol II in stabilizing Mediator occupancy at gene promoters in heat shocked cells. Previously we reported on the effect of simultaneous depletion of Taf1, TBP, or the Pol II subunit Rpb3 together with Kin28, compared to Kin28 alone, on association of Mediator with gene promoters in yeast growing in rich medium (Knoll et al. 2018). We examined the effects at two categories of gene promoters: TFIID-dominated genes, which lack consensus TATA elements, are relatively enriched for association with the Taf subunits of TFIID, and comprise $\sim 85 \%$ of 
yeast genes, including most constitutively active genes; and SAGA-dominated genes, which possess consensus TATA elements, show relatively reduced Taf association, and are enriched for inducible genes (Huisinga and Pugh 2004; Tirosh and Barkai 2008; Rhee and Pugh 2012; Donczew et al. 2020; Knoll et al. 2020). Under conditions of Kin28 depletion, Mediator association was affected by deletion of any of the PIC components examined. Concordant with the distinction between TFIID-dominated and SAGA-dominated genes, depletion of Taf1 reduced association of Med15 and Med18 to a greater degree at TFIIDdominated genes than at SAGA-dominated genes, while Rpb3 depletion reduced Mediator association strongly at all genes (Knoll et al. 2018). In contrast, simultaneous depletion of TBP and Kin28, when compared to depletion of Kin28 alone, resulted in Mediator ChIP-seq peaks shifting upstream from the proximal promoter region to UAS sites at both SAGAdominated and TFIID-dominated genes, indicating that TBP is required for transit of Mediator from its initial site of recruitment to its normally transient engagement with the proximal promoter. Similar effects are observed on comparing the effects of PIC depletion between TATA-containing, Taf1 depleted genes (enriched for SAGA-dominated genes) with TATA-less, Taf1 enriched genes (enriched for TFIID-dominated genes) (Rhee and Pugh 2012): Taf1 depletion reduces Mediator association at the latter class, and also at RP genes, much more than at TATA-containing, Taf1 enriched genes, while TBP depletion results in an upstream shift together with a decrease in intensity in the average ChIP-seq peaks for Med15 and Med18 for both categories (Figure 5A).

To determine whether Mediator association is similarly dependent on PIC components in heat shocked cells, we performed ChIP-seq against Med15 and Med18 following heat shock in yeast cells depleted for Kin28 alone or in combination with Taf1, 
TBP, or Rpb3. Somewhat surprisingly, the $\sim 300$ genes showing highest Pol II occupancy after 15 min of heat shock were relatively insensitive to depletion of either Taf1 or Rpb3, in spite of comprising similar fractions of TATA-containing and TATA-less genes (111 and 83 genes, respectively) (Figure 5B). Indeed, the TATA-containing and TATA-less subsets from this cohort behaved nearly identically: both exhibited almost no change in Med15 ChIP-seq signal upon depletion of Taf1 or Rpb3, and modest decrease in Med18 signal (Figure S4). Depletion of TBP resulted in a slight upstream shift in both Med15 and Med18 ChIP-seq signals, consistent with the effect seen in the absence of heat shock (Figure 5B). (We note that ChIP-seq signal was seen over ORF regions in these experiments. This signal appears to be artifactual, although its origin is not completely understood (Eyboulet et al. 2013; Park et al. 2013; Teytelman et al. 2013; Jeronimo and Robert 2014; Paul et al. 2015b; Grunberg et al. 2016).

Hsf1 target genes similarly showed little effect of Taf1 or Rpb3 depletion on Med15 occupancy, while Med18 ChIP-seq signal showed a small decrease (Figure 5B). Depletion of TBP led to a similar decrease in Med18 signal along with a very slight shift upstream, and to a larger decrease in Med15 signal (Figure 5B). Hsf1 target genes have been noted to be nearly equally divided among TFIID-dominated and SAGA-dominated cohorts (de Jonge et al. 2017). Finally, Mediator signal at RP genes after heat shock appeared mostly insensitive to depletion of Taf1, TBP, or Rpb3, with the exception being an increased Med18 signal upon depletion of Kin28 together with TBP (Figure 5B). This insensitivity was not surprising, inasmuch as all three of these PIC components are depleted from RP genes under heat shock conditions (Figure 1) (Reja et al. 2015; Vinayachandran et al. 2018). 


\section{Effect on Pol II and Mediator occupancy of exposure to cadmium}

The unexpected observation of persistent Mediator association with genes repressed by heat shock prompted us to examine the effect of another environmental stress on association of Mediator with gene promoters. To this end, we chose to examine the effect of exposure to $\mathrm{CdCl}_{2}$. Exposure to this toxic metal at sub-millimolar concentrations induces a rapid transcriptional response in which $\sim 150-500$ genes are induced and from 18 to 400 genes repressed (Momose and Iwahashi 2001; Cormier et al. 2010; Hosiner et al. 2014; Huang et al. 2016). Induced genes are enriched for binding sites for Hsf1, Msn2, and Msn4, and genes involved in ribosomal biogenesis are repressed by $\mathrm{CdCl}_{2}$ exposure, in common with the response to heat shock and other environmental stresses (Gasch et al. 2000; Causton et al. 2001; Hosiner et al. 2014). However, $\mathrm{CdCl}_{2}$ exposure also induces genes involved in sulfur compound metabolism (particularly the MET genes), among others, that are not involved in the heat shock response (Momose and Iwahashi 2001; Cormier et al. 2010; Hosiner et al. 2014; Huang et al. 2016), thus providing an environmental perturbation that results in a distinct but overlapping response to that elicited by heat shock.

We first tested the effect of $\mathrm{CdCl}_{2}$ exposure on Pol II association by ChIP-seq (Figure 6A). Marked induction of Pol II association was observed with the 47 genes showing the largest increase in mRNA abundance upon $\mathrm{CdCl}_{2}$ exposure (Momose and Iwahashi 2001), while association of Pol II with RP genes decreased to near baseline levels, in agreement with previous studies showing suppression of RP gene mRNA species upon $\mathrm{CdCl}_{2}$ exposure (Momose and Iwahashi 2001; Hosiner et al. 2014; Huang et al. 2016). Promoters showing at least 3-fold increase in normalized Pol II occupancy upon $\mathrm{CdCl}_{2}$ administration, and from 
the top $1000 \mathrm{Pol}$ II-occupied genes after $\mathrm{CdCl}_{2}$ exposure, overlapped strongly with those identified in microarray studies (data not shown) (Momose and Iwahashi 2001). Gene ontology analysis revealed enrichment for categories related to sulfur compound metabolism and amino acid biosynthesis, and with response to stress, as expected (Table S2). Transcription factors enriched for binding to induced genes included Hsf1, Met4, Met32, Msn2, Msn4, and Yap1, all identified previously in a microarray study (Hosiner et al. 2014), as well as Atf2, Cbf1, Met31, and Skn7 (Table S2).

We next examined the effect of $\mathrm{CdCl}_{2}$ exposure on Mediator association. ChIP-seq of Med15, from the tail module, and Med18, from the head module, under conditions of Kin28 depletion, revealed increased association at genes identified as showing increased expression upon $\mathrm{CdCl}_{2}$ administration (Figure 6B, "Momose") and at those showing increased Pol II association. Similarly to our observations of the effect of heat shock, RP genes, in spite of showing greatly reduced Pol II association upon $\mathrm{CdCl}_{2}$ exposure, exhibited persistent association of both Med15 and Med18 (Figure 6B). Also in accord with the effect of heat shock, Med18 exhibited relatively lower signal at RP genes after $\mathrm{CdCl}_{2}$ exposure than did Med15. Unlike Mediator association after heat shock, little if any upstream shift in the peaks for Med15 and Med18 at RP genes was observed after $\mathrm{CdCl}_{2}$ exposure (Figures 6B-C; compare Figures 2 and 3). To further compare heat shock and $\mathrm{CdCl}_{2}$ exposure, we examined the effect of $\mathrm{CdCl}_{2}$ exposure on Mediator and Pol II association at "UAS genes", which we earlier defined as those identified as showing Mediator ChIP signal at UAS regions under conditions of active Kin28 (Jeronimo et al. 2016). We divided these genes into those showing decreased Pol II occupancy in the presence of $\mathrm{CdCl}_{2}$ and those having unchanged or increased occupancy (Figure S5). As with UAS genes following heat shock, 
Med15 and Med18 association persisted at genes showing decreased Pol II occupancy, while increasing (Med15) or staying constant (Med18) at genes having unchanged or increased Pol II occupancy (Figure S5). However, unlike the case for heat shock, and mirroring the results for RP genes, little or no shift of Mediator to more upstream sites was observed after $\mathrm{CdCl}_{2}$ exposure. We conclude that the stresses of heat shock and $\mathrm{CdCl}_{2}$ exposure both allow continued Mediator association with repressed genes while suppressing Pol II association, but that they differ in the extent to which they allow Mediator to remain associated with gene promoters rather than UAS regions under conditions of Kin28 depletion.

\section{Discussion}

The Mediator complex was first identified as a coactivator needed to achieve activator-stimulated transcription in an in vitro system (Kornberg 2005). Consistent with this role, Mediator was shown to be recruited by activators (Kuras et al. 2003) and to facilitate looping between enhancers and promoters (Kagey et al. 2010). Recent work has added detail to this model, showing that in yeast, a single Mediator complex is first recruited to an upstream activation site and then, concomitant with its engagement with the PIC, transits to the proximal promoter region (Jeronimo et al. 2016; Petrenko et al. 2016). Association of Mediator with the proximal promoter is normally brief, but is stabilized by inhibiting Pol II escape either by inactivation or depletion of Kin28 or by loss of the Kin28 phosphorylation sites in the carboxy-terminal domain of Rpb1, the largest subunit of Pol II (Jeronimo and Robert 2014; Wong et al. 2014). Transit of Mediator from 
UAS to proximal promoter depends on TBP, and its association with the proximal promoter in the absence of Kin28 is destabilized by depletion of Taf1 or Pol II (Knoll et al. 2018).

Mediator occupancy of UAS regions of strongly induced genes, as assayed by ChIP, appears considerably stronger than at many constitutively active genes, even those expressed at high levels (Fan et al. 2006; Fan and Struhl 2009; Kim and Gross 2013). However, whether this reflects altered dynamics of Mediator with respect to the model described above has not been closely examined. In this work, we used ChIP-seq in combination with rapid depletion of Kin28 and PIC components to investigate Mediator association with activated and repressed genes following heat shock.

Consistent with previous work, we find increased association of Pol II and Mediator with genes induced by heat shock, with Mediator ChIP signal being evident even without Kin28 depletion (Figures 1-2) (Fan et al. 2006; Kim and Gross 2013; Petrenko et al. 2016; Petrenko et al. 2017). Pol II occupancy was reduced 2-3 fold upon depletion of the essential Med17 (head module) subunit of Mediator, both under both heat-shock and non-heatshock conditions, and the reduction in occupancy did not differ for targets of Hsf1 or Msn2/4 as compared to other genes expressed under heat shock conditions (Figure 1C). Deletion of tail module subunits nearly abolishes induced Pol II occupancy at heat shock responsive genes (Kim and Gross 2013), while reduction in Pol II occupancy or nascent mRNA transcription of two- to eight-fold has been reported upon acute depletion or inactivation of essential Mediator subunits in yeast (Paul et al. 2015b; Plaschka et al. 2015; Petrenko et al. 2017; Warfield et al. 2017; Bruzzone et al. 2018). Thus, genes expressed during heat shock exhibit dependence on Mediator similar to genes expressed during normal growth. The relatively uniform dependence of gene expression on Mediator 
observed in yeast is not seen in mammalian cells, as depletion of core Mediator subunits most strongly affects a subset of genes, while little effect on nascent transcription is observed at many genes (Jaeger et al. 2020).

Depletion of Kin28, which inhibits promoter escape by Pol II (Wong et al. 2014), increases Mediator ChIP signal in the absence of heat shock; the effect is gene-dependent, with, for example, RP genes exhibiting a greater increase than do Hsf1 targets (Figure 2A). Under heat shock conditions, Med15 association is not increased at Hsf1 targets by Kin28 depletion, rather exhibiting a slight shift towards the promoter (Figure 2A-B and Figure S1). These observations suggest that Mediator dynamics may be altered during heat shock, such that its residence time and consequent "ChIP-ability" may increase relative to nonheat-shock conditions. Interestingly, a genetic screen uncovered mutations in several Mediator subunits that reduced the dynamic range in the transcriptional response to heat shock (Singh et al. 2006); whether these mutations might affect dynamics of Mediator is unknown.

An unexpected finding was the persistent association of Mediator with promoters of genes repressed, and having low or negligible Pol II association, following heat shock or exposure to $\mathrm{CdCl}_{2}$ (Figures 2, 3, 4, and 6). Mediator association with UASs of genes downregulated by sulfometuron methyl treatment, which mimics amino acid starvation, was also observed in a study utilizing ChEC-seq, in which Mediator-tethered MNase is used to monitor association (Grunberg et al. 2016). Our results suggest that Mediator continues to be recruited to UASs of genes repressed by heat shock, but that recruitment of TBP and formation of the PIC is prevented by an unknown mechanism (Figure 7). The evidence in favor of this scenario is as follows: First, association of both Med15 from the tail module 
and Med18 from the head module is observed at RP genes and non-RP, "UAS genes", that are repressed following heat shock, both in the kin28-AA parent strain YFR1321 as well as the lab strain BY4741 (Figures 2-4). In kin28-AA yeast treated with rapamycin, the observed ChIP-seq signals are shifted upstream relative to their positions in the absence of heat shock, and coincide closely with Rap1 binding sites at RP genes (Figure 3A and C); Rap1 is required for Mediator recruitment to most RP genes (Ansari et al. 2009). Second, the ChIP-seq signal for Med15 does not increase at repressed RP genes upon depletion of Kin28 (Figure 2A), and neither Med15 nor Med18 ChIP signals at UAS genes that are downregulated by heat shock shift towards the proximal promoter upon depletion of Kin28 (Figure 4B). Pugh and colleagues have reported greatly reduced association of PIC components, including TBP, to genes repressed by heat shock (Vinayachandran et al. 2018). TBP appears to be required for transit of Mediator from its site of recruitment by activators to the proximal promoter (Knoll et al. 2018); thus, inhibition of PIC assembly by heat shock could prevent Mediator transit without preventing its initial recruitment, resulting in more stable association of Mediator with UAS regions at RP genes and other genes repressed by heat shock. Finally, the modest effect of depletion of TBP, Taf1, or Rpb3 on association of Med15 and Med18 with RP genes following heat shock is consistent with the lack of a PIC at these genes (Vinayachandran et al. 2018), and the engagement of Mediator at UAS regions where it would not interact with those components (Figure 5). What mechanism allows Mediator association with repressed genes following heat shock while preventing PIC formation? Two possibilities seem most likely: 1) a factor or factors actively block assembly of the PIC, with consequent failure of Mediator to transit from the UAS to the proximal promoter (Knoll et al. 2018), or 2) activators or co-activators 
that are required for PIC assembly at heat-shock-repressed genes are inactivated by heat shock, while other factors (e.g. Rap1 at RP genes) remain that are able to recruit Mediator. In either case, the mechanism must allow transcription of those genes induced or not repressed by heat shock to be active, while repressing PIC assembly at a cohort of genes that includes, but is not limited to, RP genes.

At RP genes, Rap1 binds (at 129/137 RP genes) and recruits Fhl1 and Ifh1 to activate transcription (Martin et al. 2004; Schawalder et al. 2004; Wade et al. 2004; Rudra et al. 2005), while an additional factor, Sfp1, also associates with RP gene promoters in an Fhl1-dependent fashion (like Ifh1) and contributes to recruitment of TBP and Pol II (Jorgensen et al. 2004; Marion et al. 2004; Reja et al. 2015; Albert et al. 2019). In response to stress, a signaling cascade results in translocation of Crf1 into the nucleus, which competes with Ifh1 for binding to RP genes (Martin et al. 2004). Sfp1 is also evicted from RP genes and from the nucleus in response to stress, while Rap1 and Fhl1 remain associated with RP genes; the end result is inhibition of PIC assembly and concomitant loss of transcription (Jorgensen et al. 2004; Marion et al. 2004; Martin et al. 2004; Reja et al. 2015; Vinayachandran et al. 2018). Thus, Ifh 1 and Sfp1 act as critical activators of RP gene expression. Rap1 and Fhl1 are required for recruitment of Ifh1 and Sfp1, but evidently are also able to recruit Mediator independently of Ifh1. This is surprising, as current models of transcriptional activation posit that Mediator recruitment leads directly to recruitment and assembly of the PIC. Further studies will be needed to understand the mechanism by which Mediator is recruited non-productively to genes repressed by heat shock or $\mathrm{CdCl}_{2}$, and to establish whether some "activators" may recruit Mediator but nonetheless be insufficient to facilitate PIC assembly and activate transcription. 
The dynamics of Mediator recruitment and participation in PIC assembly have only recently begun to be appreciated, and thus far only in the model organism Saccharomyces cerevisiae (Jeronimo and Robert 2017). It is clear that in budding yeast, these dynamics vary in a gene-dependent fashion, as Mediator occupancy varies greatly at UAS regions and does not correlate with transcriptional output (Jeronimo and Robert 2014; Paul et al. 2015b; Grunberg et al. 2016). The work reported here shows that Mediator dynamics, including its association with repressed genes, also can vary in a condition-dependent fashion. Mediator itself is affected in its post-translational modifications and its composition by alterations in environment such as osmotic shock and the transition to stationary phase (Holstege et al. 1998; Miller et al. 2012); whether and how Mediator dynamics are affected by such alterations is currently obscure. Finally, in metazoan organisms, Mediator associates with enhancers that are sometimes many kilobases removed from the sites of PIC assembly at which Mediator participates via loop formation (Kagey et al. 2010); the dynamics of such Mediator-dependent loop formation, and the variables that influence those dynamics, remain an area for future investigation.

\section{Methods}

Yeast strains and growth

Yeast strains used in this study are listed in Table S3. Epitope tags were introduced into BY4741 to generate strains RMYDS1 and RMYDS2, and into YFR1321 to generate RMYDS10, by PCR amplification from strains containing the tagged protein and selectable marker, followed by transformation and selection (Hill et al. 1991; Longtine et al. 1998); strains were verified by PCR and ChIP. For simplicity, epitope-tagged strains are referred to 
by the parent strain names in the text and figures; for example, "BY4741" refers also to RMYDS1, which harbors the med15-myc allele. Cultures were grown in yeast peptone dextrose (YPD) medium (1\% bacto-yeast extract, 2\% bacto-peptone extract, 2\% glucose). For Rap1 ChIP in wild type and rap1-ts yeast (Li et al. 2011), cultures were shifted to $37^{\circ} \mathrm{C}$ for $1 \mathrm{hr}$ before cross-linking and ChIP and processed as described previously (Paul et al. 2015a). Anchor away experiments were carried out as reported previously (Knoll et al. 2018). In brief, yeast were grown to $0.6-0.8 \mathrm{OD}_{600}$ at $30^{\circ} \mathrm{C}$ and then rapamycin (LC Laboratories, Woburn, MA, USA) was added from a $1 \mathrm{mg} / \mathrm{ml}$ stock solution in ethanol to a final concentration of $1 \mu \mathrm{g} / \mathrm{ml}$. One hour after rapamycin treatment, one-third volume of prewarmed media $\left(13 \mathrm{ml}\right.$ at $57^{\circ} \mathrm{C}$ added to $39 \mathrm{ml}$ culture for a final temperature of $\left.37^{\circ} \mathrm{C}\right)$, or one-third volume of media at $30^{\circ} \mathrm{C}$ for non-heat-shocked cells, was added to each flask and flasks were immediately placed at $37^{\circ} \mathrm{C}$ (or at $30^{\circ} \mathrm{C}$ for non-heat-shocked cells) in a shaking incubator. Both kin28-AA yeast and the parent strain YFR1321 were treated with rapamycin in all experiments unless indicated otherwise. Samples were incubated for 15 min or 30 min prior to cross-linking with formaldehyde. Similarly, for $\mathrm{CdCl}_{2}$ treatment, yeast cultures were grown and treated with rapamycin as described above. After 1 hour of rapamycin treatment, $\mathrm{CdCl}_{2}$ was added from a $1 \mathrm{M}$ stock solution to a final concentration of $0.5 \mathrm{mM}$ and incubated for another hour before crosslinking.

ChIP-seq

ChIP was performed as described previously (Knoll et al. 2018). For IP, $600 \mu \mathrm{L}$ of WCE was incubated overnight at $4{ }^{\circ} \mathrm{C}$ with $10 \mu \mathrm{g}$ of monoclonal RNA Poll antibody (Biolegend, USA), $2 \mu \mathrm{g}$ of anti-myc antibody (Sigma), or $2 \mu \mathrm{g}$ of anti-Rap1 antibody (Santa 
Cruz, USA). Sixty microliters of WCE was used as Input control. Immunoprecipitated DNA was purified using $40 \mu \mathrm{L}$ of protein $\mathrm{A}$ or $\mathrm{G}$ beads (Amersham/GE) with gentle agitation at $4^{\circ} \mathrm{C}$ for $90 \mathrm{~min}$, and cleanup and purification performed as described previously (Knoll et al. 2018).

Libraries were prepared for sequencing using the NEBNext Ultra II library preparation kit (New England Biolabs, USA) according to manufacturer's protocol and barcoded using NEXTflex barcodes (BIOO Scientific, Austin, TX, USA) or NEBNext Multiplex Oligos for Illumina. Purification and the size selection step were performed on barcoded libraries by isolating fragment sizes between 200 and 500 bp by using AMPureXP beads (Beckman Coulter, USA); size selection was confirmed by Bioanalyzer. Sequencing was performed at the Illumina NextSeq platform at the Wadsworth Center, New York State Department of Health (Albany, NY, USA) or at the Carolina Center for the Genome Sciences, University of North Carolina at Chapel Hill for Rap1 ChIP-seq. ChIP-seq experiments are summarized, and corresponding deposited files indicated, in Table S4. We also used previously published ChIP-seq data deposited at the NCBI Short Read Archive under accession number PRJNA413080 (Knoll et al. 2018).

\section{ChIP-seq analysis}

Unfiltered sequencing reads were aligned to the $S$. cerevisiae reference genome (Saccer3) using bwa (Seoighe and Wolfe 1999). Up to 1 mismatch was allowed for each aligned read. Reads mapping to multiple sites were retained to allow evaluation of associations with non-unique sequences (Seoighe and Wolfe 1999) and paired end reads were removed, with the exception of the Rap1 ChIP-seq data, which yielded single-end 
reads and for which duplicate reads were retained. Calculation of coverage, as shown in heat maps and line graphs, was preceded by library size normalization, and was performed with the "chipseq" and "GenomicRanges" packages in BioConductor (Gentleman et al. 2004). Alternatively, reads were aligned and analysis conducted using the Galaxy platform (Goecks et al. 2010) and Excel. For metagene analysis, including heat maps, we subtracted reads from an input control (strain YFR1321, the parent strain to kin28-AA yeast (KHW127), grown in the absence of rapamycin); for Rap1, reads from the rap1-2 ts mutant, which has greatly reduced binding at $37^{\circ} \mathrm{C}$ (Drazinic et al. 1996; Ganapathi et al. 2011), were subtracted from the wild type strain also grown at $37^{\circ} \mathrm{C}$ for one hour. Pol II occupancy was determined as read depth over ORFs, and Mediator occupancy was determined as normalized read depth over the 300 bp upstream of TSS using BedCov in SamTools (Li et al. 2009). Clustering analysis (Figure 1) was performed using Cluster and Treeview (Eisen et al. 1998). The 1000 genes having highest Pol II occupancy, normalized to gene length, were obtained using BedCov to obtain read depth over coding sequences using Pol II ChIP-seq data (strain BY4741 grown at $30^{\circ} \mathrm{C}$ in YPD medium) (Paul et al. 2015b; Knoll et al. 2018). Genes designated as SAGA-dominated and TFIID-dominated were obtained from (Huisinga and Pugh 2004), and genes designated as containing or not containing a consensus TATA element, and being Taf1-enriched or Taf1-depleted, were obtained from (Rhee and Pugh 2012). Targets of Hsf1 were defined as those genes that are not activated by heat shock if Hsf1 is depleted (Pincus et al. 2018; Tye et al. 2019), Msn2/4 targets were defined in (Solis et al. 2016), and $\mathrm{CdCl} 2$ induced genes were those identified in Table 3 of (Momose and Iwahashi 2001). Occupancy profiles were normalized for read depth and generated using the Integrative Genomics Viewer (Robinson et al. 2011). Gene ontology analysis was 
performed using the Generic Gene Ontology Term Finder (https://go.princeton.edu/cgibin/GOTermFinder/GOTermFinder) (Boyle et al. 2004). Hypergeometric test p-values were calculated using the online calculator at http://www.alewand.de/stattab/tabdiske.htm.

\section{Data access}

ChIP-seq reads have been deposited in the NCBI Short Read Archive (https://www.ncbi.nlm.nih.gov/sra) under project number PRJNA657372.

\section{Acknowledgements}

We thank Francois Robert and Kevin Struhl for generously providing yeast strains, Jason Lieb and Colin Lickwar for help in ChIP-seq of Rap1, and Elisabeth Knoll for helpful discussions. We gratefully acknowledge help from the Wadsworth Center Applied Genomics Technology and Tissue Culture and Media Cores. This work was supported by the National Science Foundation (MCB1516839 to RHM) and in part by the NIH Intramural Research Program at the National Library of Medicine (ZIZ and DL).

\section{Disclosure declaration}

No conflicts of interest declared.

\section{References}

Albert B, Tomassetti S, Gloor Y, Dilg D, Mattarocci S, Kubik S, Hafner L, Shore D. 2019. Sfp1 regulates transcriptional networks driving cell growth and division through multiple promoter-binding modes. Genes Dev 33: 288-293.

Ansari SA, He Q, Morse RH. 2009. Mediator complex association with constitutively transcribed genes in yeast. P Natl Acad Sci USA 106: 16734-16739. 
Boyle EI, Weng S, Gollub J, Jin H, Botstein D, Cherry JM, Sherlock G. 2004. GO::TermFinder-open source software for accessing Gene Ontology information and finding significantly enriched Gene Ontology terms associated with a list of genes. Bioinformatics 20: 3710-3715.

Bruzzone MJ, Grunberg S, Kubik S, Zentner GE, Shore D. 2018. Distinct patterns of histone acetyltransferase and Mediator deployment at yeast protein-coding genes. Genes Dev 32: 1252-1265.

Causton HC, Ren B, Koh SS, Harbison CT, Kanin E, Jennings EG, Lee TI, True HL, Lander ES, Young RA. 2001. Remodeling of yeast genome expression in response to environmental changes. Mol Biol Cell 12: 323-337.

Cormier L, Barbey R, Kuras L. 2010. Transcriptional plasticity through differential assembly of a multiprotein activation complex. Nucleic Acids Res 38: 4998-5014.

de Jonge WJ, O'Duibhir E, Lijnzaad P, van Leenen D, Groot Koerkamp MJ, Kemmeren P, Holstege FC. 2017. Molecular mechanisms that distinguish TFIID housekeeping from regulatable SAGA promoters. EMBO J 36: 274-290.

Donczew R, Warfield L, Pacheco D, Erijman A, Hahn S. 2020. Two roles for the yeast transcription coactivator SAGA and a set of genes redundantly regulated by TFIID and SAGA. Elife 9.

Drazinic CM, Smerage JB, Lopez MC, Baker HV. 1996. Activation mechanism of the multifunctional transcription factor repressor-activator protein 1 (Rap1p). Mol Cell Biol 16: 3187-3196.

Eisen MB, Spellman PT, Brown PO, Botstein D. 1998. Cluster analysis and display of genome-wide expression patterns. Proc Natl Acad Sci U S A 95: 14863-14868.

Eyboulet F, Cibot C, Eychenne T, Neil H, Alibert O, Werner M, Soutourina J. 2013. Mediator links transcription and DNA repair by facilitating Rad2/XPG recruitment. Genes Dev 27: 2549-2562.

Fan X, Chou DM, Struhl K. 2006. Activator-specific recruitment of Mediator in vivo. Nat Struct Mol Biol 13: 117-120.

Fan X, Struhl K. 2009. Where does mediator bind in vivo? PLoS One 4: e5029.

Ganapathi M, Palumbo MJ, Ansari SA, He Q, Tsui K, Nislow C, Morse RH. 2011. Extensive role of the general regulatory factors, Abf1 and Rap1, in determining genome-wide chromatin structure in budding yeast. Nucleic Acids Res 39: 2032-2044.

Gasch AP, Spellman PT, Kao CM, Carmel-Harel O, Eisen MB, Storz G, Botstein D, Brown PO. 2000. Genomic expression programs in the response of yeast cells to environmental changes. Mol Biol Cell 11: 4241-4257.

Gentleman RC, Carey VJ, Bates DM, Bolstad B, Dettling M, Dudoit S, Ellis B, Gautier L, Ge Y, Gentry J et al. 2004. Bioconductor: open software development for computational biology and bioinformatics. Genome Biol 5: R80.

Goecks J, Nekrutenko A, Taylor J. 2010. Galaxy: a comprehensive approach for supporting accessible, reproducible, and transparent computational research in the life sciences. Genome Biol 11: R86.

Gross DS, English KE, Collins KW, Lee SW. 1990. Genomic footprinting of the yeast HSP82 promoter reveals marked distortion of the DNA helix and constitutive occupancy of heat shock and TATA elements. J Mol Biol 216: 611-631. 
Grunberg S, Henikoff S, Hahn S, Zentner GE. 2016. Mediator binding to UASs is broadly uncoupled from transcription and cooperative with TFIID recruitment to promoters. EMBO J 35: 2435-2446.

Haruki H, Nishikawa J, Laemmli UK. 2008. The anchor-away technique: rapid, conditional establishment of yeast mutant phenotypes. Mol Cell 31: 925-932.

Hill J, Donald KA, Griffiths DE, Donald G. 1991. DMSO-enhanced whole cell yeast transformation [published erratum appears in Nucleic Acids Res 1991 Dec 11;19(23):6688]. Nucleic Acids Res 19: 5791.

Holstege FC, Jennings EG, Wyrick JJ, Lee TI, Hengartner CJ, Green MR, Golub TR, Lander ES, Young RA. 1998. Dissecting the regulatory circuitry of a eukaryotic genome. Cell 95: 717-728.

Hosiner D, Gerber S, Lichtenberg-Frate H, Glaser W, Schuller C, Klipp E. 2014. Impact of acute metal stress in Saccharomyces cerevisiae. PLoS One 9: e83330.

Huang X, Li Y, Pan J, Li M, Lai Y, Gao J, Li X. 2016. RNA-Seq identifies redox balance related gene expression alterations under acute cadmium exposure in yeast. Environ Microbiol Rep 8: 1038-1047.

Huisinga KL, Pugh BF. 2004. A genome-wide housekeeping role for TFIID and a highly regulated stress-related role for SAGA in Saccharomyces cerevisiae. Mol Cell 13: 573-585.

Jaeger MG, Schwalb B, Mackowiak SD, Velychko T, Hanzl A, Imrichova H, Brand M, Agerer B, Chorn S, Nabet B et al. 2020. Selective Mediator dependence of cell-type-specifying transcription. Nat Genet 52: 719-727.

Jeronimo C, Langelier MF, Bataille AR, Pascal JM, Pugh BF, Robert F. 2016. Tail and Kinase Modules Differently Regulate Core Mediator Recruitment and Function In Vivo. Mol Cell 64: 455-466.

Jeronimo C, Robert F. 2014. Kin28 regulates the transient association of Mediator with core promoters. Nat Struct Mol Biol 21: 449-455.

Jeronimo C, Robert F. 2017. The Mediator Complex: At the Nexus of RNA Polymerase II Transcription. Trends Cell Biol 27: 765-783.

Jorgensen P, Rupes I, Sharom JR, Schneper L, Broach JR, Tyers M. 2004. A dynamic transcriptional network communicates growth potential to ribosome synthesis and critical cell size. Genes Dev 18: 2491-2505.

Kagey MH, Newman JJ, Bilodeau S, Zhan Y, Orlando DA, van Berkum NL, Ebmeier CC, Goossens J, Rahl PB, Levine SS et al. 2010. Mediator and cohesin connect gene expression and chromatin architecture. Nature 467: 430-435.

Kim S, Gross DS. 2013. Mediator recruitment to heat shock genes requires dual Hsf1 activation domains and mediator tail subunits Med15 and Med16. J Biol Chem 288: 12197-12213.

Kim TS, Liu CL, Yassour M, Holik J, Friedman N, Buratowski S, Rando OJ. 2010. RNA polymerase mapping during stress responses reveals widespread nonproductive transcription in yeast. Genome Biol 11: R75.

Knight B, Kubik S, Ghosh B, Bruzzone MJ, Geertz M, Martin V, Denervaud N, Jacquet P, Ozkan B, Rougemont J et al. 2014. Two distinct promoter architectures centered on dynamic nucleosomes control ribosomal protein gene transcription. Genes Dev 28: 1695-1709. 
Knoll ER, Zhu ZI, Sarkar D, Landsman D, Morse RH. 2018. Role of the pre-initiation complex in Mediator recruitment and dynamics. Elife 7.

Knoll ER, Zhu ZI, Sarkar D, Landsman D, Morse RH. 2020. Kin28 depletion increases association of TFIID subunits Taf1 and Taf4 with promoters in Saccharomyces cerevisiae. Nucleic Acids Res 48: 4244-4255.

Kornberg RD. 2005. Mediator and the mechanism of transcriptional activation. Trends Biochem Sci 30: 235-239.

Kuras L, Borggrefe T, Kornberg RD. 2003. Association of the Mediator complex with enhancers of active genes. Proc Natl Acad Sci U S A 100: 13887-13891.

Lee D, Lis JT. 1998. Transcriptional activation independent of TFIIH kinase and the RNA polymerase II mediator in vivo. Nature 393: 389-392.

Li H, Handsaker B, Wysoker A, Fennell T, Ruan J, Homer N, Marth G, Abecasis G, Durbin R, Genome Project Data Processing S. 2009. The Sequence Alignment/Map format and SAMtools. Bioinformatics 25: 2078-2079.

Li Z, Vizeacoumar FJ, Bahr S, Li J, Warringer J, Vizeacoumar FS, Min R, Vandersluis B, Bellay J, Devit M et al. 2011. Systematic exploration of essential yeast gene function with temperature-sensitive mutants. Nat Biotechnol 29: 361-367.

Lieb JD, Liu X, Botstein D, Brown PO. 2001. Promoter-specific binding of Rap1 revealed by genome-wide maps of protein-DNA association. Nat Genet 28: 327-334.

Longtine MS, McKenzie A, 3rd, Demarini DJ, Shah NG, Wach A, Brachat A, Philippsen P, Pringle JR. 1998. Additional modules for versatile and economical PCR-based gene deletion and modification in Saccharomyces cerevisiae. Yeast 14: 953-961.

Marion RM, Regev A, Segal E, Barash Y, Koller D, Friedman N, O'Shea EK. 2004. Sfp1 is a stress- and nutrient-sensitive regulator of ribosomal protein gene expression. Proc Natl Acad Sci U S A 101: 14315-14322.

Martin DE, Soulard A, Hall MN. 2004. TOR regulates ribosomal protein gene expression via PKA and the Forkhead transcription factor FHL1. Cell 119: 969-979.

Mayer C, Grummt I. 2006. Ribosome biogenesis and cell growth: mTOR coordinates transcription by all three classes of nuclear RNA polymerases. Oncogene 25: 63846391.

McKinlay A, Araya CL, Fields S. 2011. Genome-Wide Analysis of Nascent Transcription in Saccharomyces cerevisiae. G3 (Bethesda) 1: 549-558.

McNeil JB, Agah H, Bentley D. 1998. Activated transcription independent of the RNA polymerase II holoenzyme in budding yeast. Genes Dev 12: 2510-2521.

Mencia M, Moqtaderi Z, Geisberg JV, Kuras L, Struhl K. 2002. Activator-specific recruitment of TFIID and regulation of ribosomal protein genes in yeast. Mol Cell 9: 823-833.

Miller C, Matic I, Maier K, Schwalb B, Roether S, Straesser K, Tresch A, Mann M, Cramer P. 2012. Mediator phosphorylation prevents stress response transcription during nonstress conditions. J Biol Chem doi:M112.430140 [pii]

10.1074/jbc.M112.430140.

Momose Y, Iwahashi H. 2001. Bioassay of cadmium using a DNA microarray: genome-wide expression patterns of Saccharomyces cerevisiae response to cadmium. Environ Toxicol Chem 20: 2353-2360.

Morris RT, O'Connor TR, Wyrick JJ. 2010. Ceres: software for the integrated analysis of transcription factor binding sites and nucleosome positions in S. cerevisiae. Bioinformatics. 
Park D, Lee Y, Bhupindersingh G, Iyer VR. 2013. Widespread Misinterpretable ChIP-seq Bias in Yeast. PLoS One 8: e83506.

Paul E, Tirosh I, Lai W, Buck MJ, Palumbo MJ, Morse RH. 2015a. Chromatin mediation of a transcriptional memory effect in yeast. G3 (Bethesda) 5: 829-838.

Paul E, Zhu ZI, Landsman D, Morse RH. 2015b. Genome-wide association of mediator and RNA polymerase II in wild-type and mediator mutant yeast. Mol Cell Biol 35: 331342.

Petrenko N, Jin Y, Wong KH, Struhl K. 2016. Mediator Undergoes a Compositional Change during Transcriptional Activation. Mol Cell 64: 443-454.

Petrenko N, Jin Y, Wong KH, Struhl K. 2017. Evidence that Mediator is essential for Pol II transcription, but is not a required component of the preinitiation complex in vivo. Elife 6.

Pincus D, Anandhakumar J, Thiru P, Guertin MJ, Erkine AM, Gross DS. 2018. Genetic and epigenetic determinants establish a continuum of Hsf1 occupancy and activity across the yeast genome. Mol Biol Cell 29: 3168-3182.

Plaschka C, Lariviere L, Wenzeck L, Seizl M, Hemann M, Tegunov D, Petrotchenko EV, Borchers CH, Baumeister W, Herzog F et al. 2015. Architecture of the RNA polymerase II-Mediator core initiation complex. Nature 518: 376-380.

Plaschka C, Nozawa K, Cramer P. 2016. Mediator Architecture and RNA Polymerase II Interaction. J Mol Biol 428: 2569-2574.

Reja R, Vinayachandran V, Ghosh S, Pugh BF. 2015. Molecular mechanisms of ribosomal protein gene coregulation. Genes Dev 29: 1942-1954.

Rhee HS, Pugh BF. 2012. Genome-wide structure and organization of eukaryotic preinitiation complexes. Nature 483: 295-301.

Robinson JT, Thorvaldsdottir H, Winckler W, Guttman M, Lander ES, Getz G, Mesirov JP. 2011. Integrative genomics viewer. Nat Biotechnol 29: 24-26.

Rudra D, Zhao Y, Warner JR. 2005. Central role of Ifh1p-Fhl1p interaction in the synthesis of yeast ribosomal proteins. Embo J 24: 533-542.

Schawalder SB, Kabani M, Howald I, Choudhury U, Werner M, Shore D. 2004. Growthregulated recruitment of the essential yeast ribosomal protein gene activator Ifh1. Nature 432: 1058-1061.

Schier AC, Taatjes DJ. 2020. Structure and mechanism of the RNA polymerase II transcription machinery. Genes Dev 34: 465-488.

Seoighe C, Wolfe KH. 1999. Updated map of duplicated regions in the yeast genome. Gene 238: 253-261.

Singh H, Erkine AM, Kremer SB, Duttweiler HM, Davis DA, Iqbal J, Gross RR, Gross DS. 2006. A functional module of yeast mediator that governs the dynamic range of heat-shock gene expression. Genetics 172: 2169-2184.

Solis EJ, Pandey JP, Zheng X, Jin DX, Gupta PB, Airoldi EM, Pincus D, Denic V. 2016. Defining the Essential Function of Yeast Hsf1 Reveals a Compact Transcriptional Program for Maintaining Eukaryotic Proteostasis. Mol Cell 63: 60-71.

Soutourina J. 2018. Transcription regulation by the Mediator complex. Nat Rev Mol Cell Biol 19: $262-274$.

Teytelman L, Thurtle DM, Rine J, van Oudenaarden A. 2013. Highly expressed loci are vulnerable to misleading ChIP localization of multiple unrelated proteins. Proc Natl Acad Sci U S A 110: 18602-18607. 
Tirosh I, Barkai N. 2008. Two strategies for gene regulation by promoter nucleosomes. Genome Res 18: 1084-1091.

Tsai KL, Tomomori-Sato C, Sato S, Conaway RC, Conaway JW, Asturias FJ. 2014. Subunit architecture and functional modular rearrangements of the transcriptional mediator complex. Cell 157: 1430-1444.

Tye BW, Commins N, Ryazanova LV, Wuhr M, Springer M, Pincus D, Churchman LS. 2019. Proteotoxicity from aberrant ribosome biogenesis compromises cell fitness. Elife 8.

van de Peppel J, Kettelarij N, van Bakel H, Kockelkorn TT, van Leenen D, Holstege FC. 2005. Mediator expression profiling epistasis reveals a signal transduction pathway with antagonistic submodules and highly specific downstream targets. Mol Cell 19: 511522.

Vinayachandran V, Reja R, Rossi MJ, Park B, Rieber L, Mittal C, Mahony S, Pugh BF. 2018. Widespread and precise reprogramming of yeast protein-genome interactions in response to heat shock. Genome Res doi:10.1101/gr.226761.117.

Wade JT, Hall DB, Struhl K. 2004. The transcription factor Ifh1 is a key regulator of yeast ribosomal protein genes. Nature 432: 1054-1058.

Wang X, Sun Q, Ding Z, Ji J, Wang J, Kong X, Yang J, Cai G. 2014. Redefining the modular organization of the core Mediator complex. Cell Res 24: 796-808.

Warfield L, Ramachandran S, Baptista T, Devys D, Tora L, Hahn S. 2017. Transcription of Nearly All Yeast RNA Polymerase II-Transcribed Genes Is Dependent on Transcription Factor TFIID. Mol Cell 68: 118-129 e115.

Warner JR. 1999. The economics of ribosome biosynthesis in yeast. Trends Biochem Sci 24: 437-440.

Wong KH, Jin Y, Struhl K. 2014. TFIIH phosphorylation of the Pol II CTD stimulates mediator dissociation from the preinitiation complex and promoter escape. Mol Cell 54: 601-612.

Zeevi D, Sharon E, Lotan-Pompan M, Lubling Y, Shipony Z, Raveh-Sadka T, Keren L, Levo M, Weinberger A, Segal E. 2011. Compensation for differences in gene copy number among yeast ribosomal proteins is encoded within their promoters. Genome Res 21: 2114-2128.

\section{Figure Legends}

Figure 1. Pol II recruitment following heat shock. (A) Heat maps and line graphs depicting normalized Pol II occupancy in YFR1321 cells before and after 15 min heat shock at 42 Hsf1 targets and 213 Msn2-4 targets (see Methods) and 137 RP genes. (B) K-means clustering $(\mathrm{K}=6)$ was performed for the ratio of Pol II occupancy (normalized for gene length) before and after heat shock, using the 1000 genes having highest Pol II occupancy under non-heat-shock conditions plus the 300 gene having highest occupancy after heat 
shock (1179 ORFs, due to overlap between the two sets). Enrichment for TFs in individual clusters, as shown, derived from CERES (Morris et al. 2010). (C) Box and whisker plots depicting ratios of Pol II occupancy (normalized for gene length) with and without heat shock, as indicated, in the med17-AA strain (YFR1544) and parent strain (YFR1321). Left panel depicts replicate experiments examining the 886 genes with highest Pol II occupancy in the absence of heat shock (three outliers with ratios $>8$ in replicate 1 were removed for clarity) and 294 genes having highest occupancy after heat shock. Right panel depicts the 294 genes having highest Pol II occupancy after heat shock, divided into Hsf1 targets (34 genes), Msn2-4 targets (48 genes), and other genes (212 genes).

Figure 2. Effect of heat shock on Mediator association. (A) Heat maps and line graphs depicting normalized occupancy of the Mediator tail module subunit, Med15, in kin28AA yeast treated with rapamycin ("kin28AAR") and the parent strain YFR1321, also treated with rapamycin, before and after 15 min heat shock, at 42 Hsf1 targets and 213 Msn2-4 targets (see Methods) and 137 RP genes. (B) Box and whisker plots showing the ratios of Med15 occupancy with and without Kin28 depletion for the $\sim 300$ genes showing highest Med15 occupancy in Kin28-depleted cells without or with heat shock; ratios are also shown for RP genes (137 genes), and for Hsf1 targets (42 genes) and Msn2-4 targets (213 genes) in heat-shocked cells. The p-value for comparison of the ratios for the top 300 genes with and without heat shock was calculated using the Wilcoxon rank sum test. (C) Browser scans showing Med15 occupancy upstream of BAP2 and UBI4 in kin28AA yeast and the parent strain YFR1321, both treated with rapamycin, with and without heat shock. UBI4 is a target of Hsf1, while BAP2 is not a target of Hsf1 or Msn2-4. Scale, in reads per million 
mapped reads, is indicated for each scan. (D) Heat maps and line graphs depicting occupancy of the Mediator tail module subunit, Med15, in BY4741 yeast, kin28AA yeast treated with rapamycin ("kin28AAR") and the parent strain YFR1321, also treated with rapamycin, in the absence of heat shock, at 498 "UAS” genes (see text) and 137 RP genes.

Figure 3. Mediator association persists at RP genes after heat shock. (A) Browser scans showing normalized occupancy of Med15 upstream of RPL42A and RPS4B in kin28AA yeast and the parent strain YFR1321, both treated with rapamycin, with and without heat shock (top four scans) or Rap1 in strain BY4741 (bottom scan). Scale, in reads per million mapped reads, is indicated for each scan. (B) Heat maps and line graphs depicting normalized occupancy of the Mediator head module subunit, Med18, in kin28AA yeast in the presence of rapamycin, with and without heat shock, as 42 Hsf1 targets and 137 RP genes. (C) Heat maps and line graphs depicting normalized occupancy of Mediator subunits Med15 (tail) and Med18 (head), and Rap1, at Hsf1 target genes and RP genes in BY4741. The baseline for the Rap1 line graph was rescaled to align with the other line graphs. The signal observed at ORF regions (seen at Hsf1 targets under heat shock conditions, and at RP genes under non-heat-shocked conditions) is a ChIP artifact frequently observed at highly transcribed ORFs (Eyboulet et al. 2013; Park et al. 2013; Teytelman et al. 2013; Jeronimo and Robert 2014; Knoll et al. 2020).

Figure 4. Mediator association at non-RP genes repressed by heat shock. (A) Heat maps and line graphs showing normalized occupany of Pol II and Med15 at UAS genes (see text) having Pol II occupancy decreased by at least 2-fold upon heat shock ("UAS down") or 
having Pol II occupancy unchanged or increased upon heat shock (“UAS not down”) in YFR1321, the parent strain to kin28-AA yeast, with and without heat shock. (B) Heat maps and line graphs showing normalized occupany of Med15 (tail) and Med18 (head) at "UAS down" and "UAS not down" genes in kin28-AA yeast treated with rapamycin, with and without heat shock. (C) Browser scans showing Med15 and Pol II occupancy at ILV1 and TEF1 genes in kin28-AA yeast ("+ Kin28”) or the parent strain YFR1321 ("- Kin28”), both treated with rapamycin, with or without heat shock. Scale, in reads per million mapped reads, is indicated for each scan. Note that Pol II occupancy is reduced at both genes upon heat shock; the vertical dashed lines emphasize the shift of the Med15 peak towards the promoters only when Kin28 is depleted in the absence of heat shock.

Figure 5. Effect of depleting PIC components on Mediator association with gene promoters. (A) Heat maps and line graphs showing normalized occupancy of Med15 (tail) and Med18 (head) at TATA-containing, Taf1 depleted promoters from the 1000 genes with highest Pol II occupancy (228 genes), TATA-less, Taf1 enriched genes excluding RP genes from the 1000 genes with highest Pol II occupancy (330 genes), and 137 RP genes after depletion of Kin28 alone or together with Taf1, TBP, or Rpb3, as indicated. (B) Heat maps and line graphs showing normalized occupancy of Med15 (tail) and Med18 (head) at the 300 genes with highest Pol II occupancy after heat shock, 42 Hsf1 targets, and 137 RP genes after depletion of Kin28 alone or together with Taf1, TBP, or Rpb3, as indicated, without or with 15 min heat shock, as indicated. 
Figure 6. Effect on Pol II and Mediator occupancy of $\mathrm{CdCl}_{2}$ exposure. (A) Heat maps and line graphs showing normalized occupancy of Pol II at 47 strongly induced genes (Momose and Iwahashi 2001) and 137 RP genes in the anchor away parent strain, YFR1321. (B) Heat maps and line graphs showing normalized occupancy by Med15 (tail module) and Med18 (head module) at 47 strongly induced genes, the 500 genes having the highest ratio of induced to uninduced Pol II occupancy and 137 RP genes. (C) Browser scans showing Med15 occupancy in reads per million mapped reads after Kin28 depletion in unstressed cells, cells exposed to $\mathrm{CdCl}_{2}$, and after 15 min of heat shock, as indicated, at the RPS4B and RPL42A loci.

Figure 7. Cartoon of Mediator recruitment and transcriptional activation. Under normal growth conditions, Mediator is recruited via its tail module by an activator bound to a UAS, and in turn recruits components of the PIC, with TBP being required for Mediator transit from UAS to the proximal promoter, which may or may not possess a consensus TATA element. Association of Mediator with the proximal promoter is normally transient, being rapidly lost upon Kin28-dependent promoter escape by Pol II, but is stabilized by depletion or inactivation of Kin28. Heat shock or other stress (such as $\mathrm{CdCl}_{2}$ exposure) prevents PIC assembly and transit of Mediator to the proximal promoter through an unknown mechanism. 
A

Pol II ChIP

${ }_{16}$ Hsf1 targets Msn2-4 RP genes

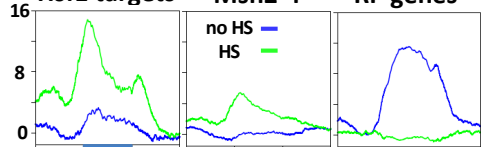

$0-1 \mathrm{mb}$

no HS
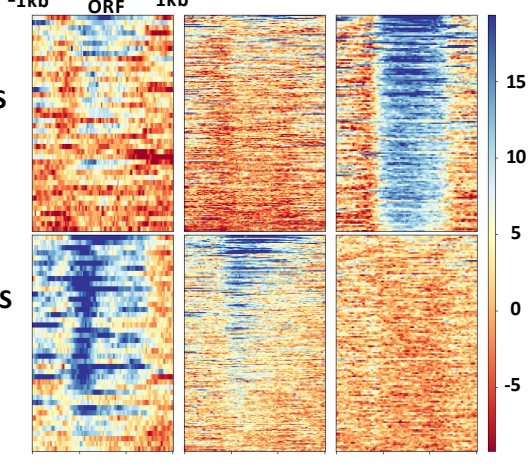

15 min HS

C

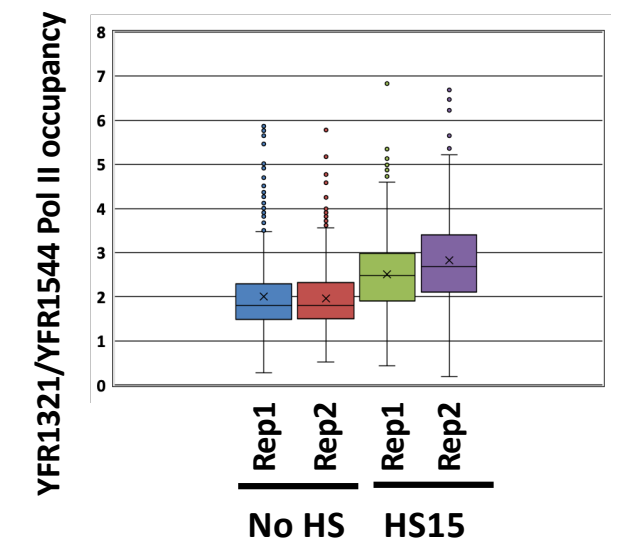

B

Pol II ChIP ratios heat shock/non-heat shock

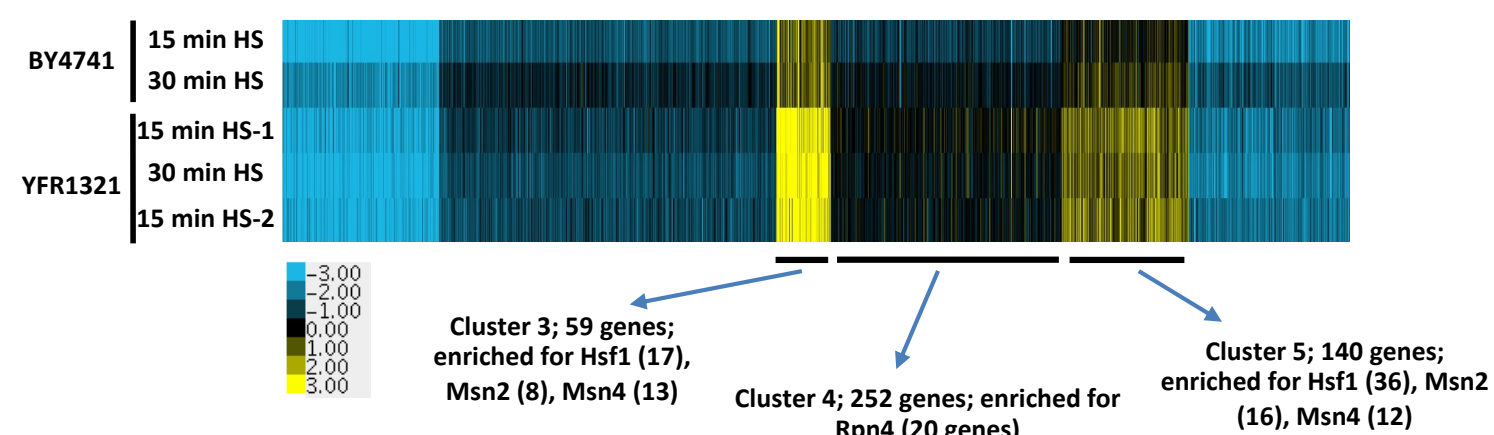

(16), Msn4 (12)

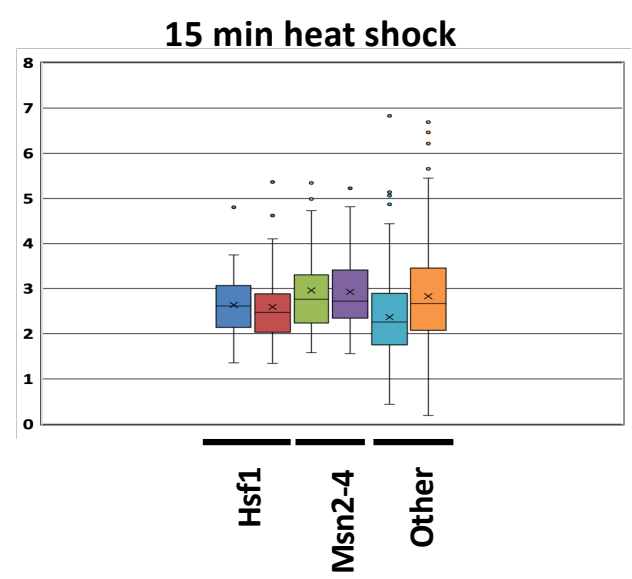

Figure 1 


\section{Figure 2}

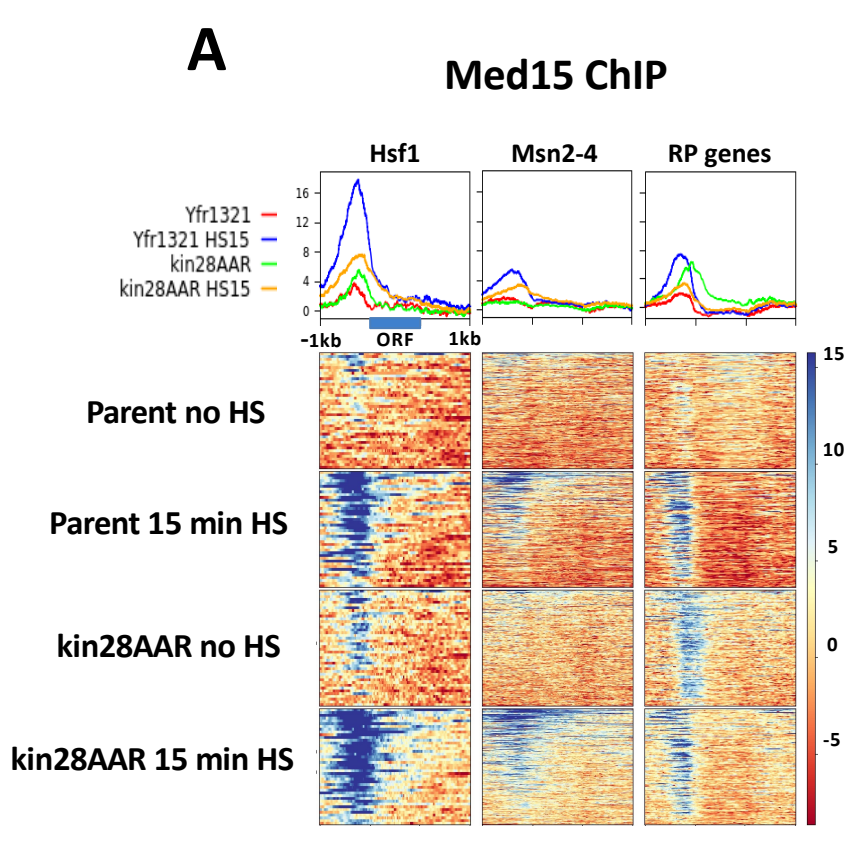

\section{B Med15 promoter occupancy ratios}

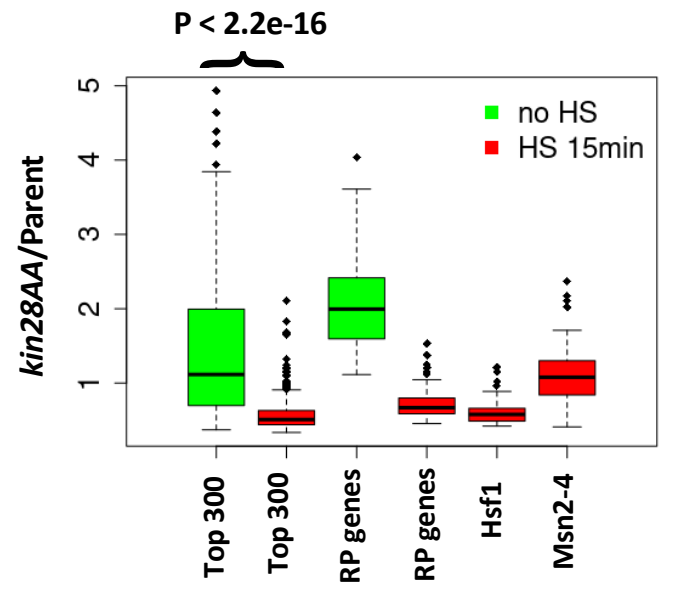

\section{C}

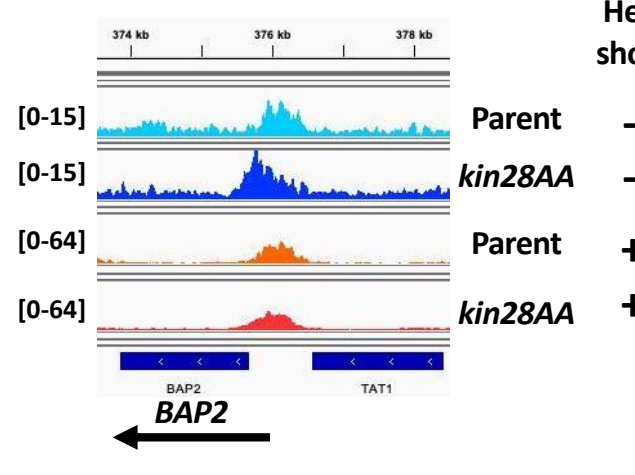

\section{Heat}

shock

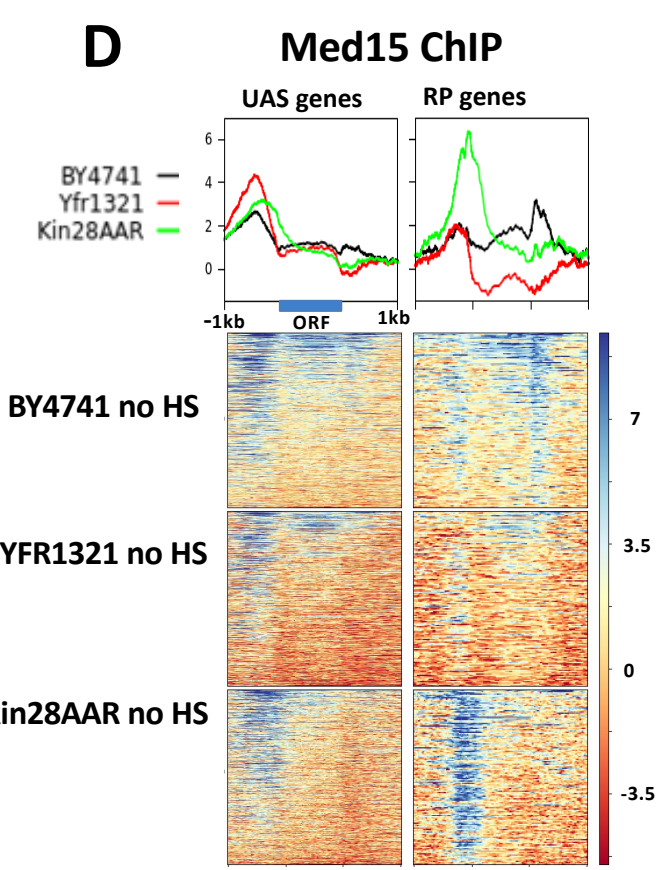

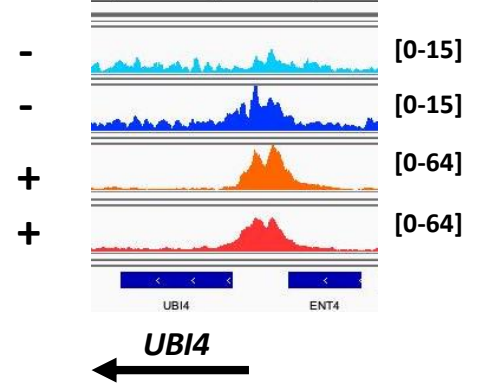




\section{Figure 3}

A

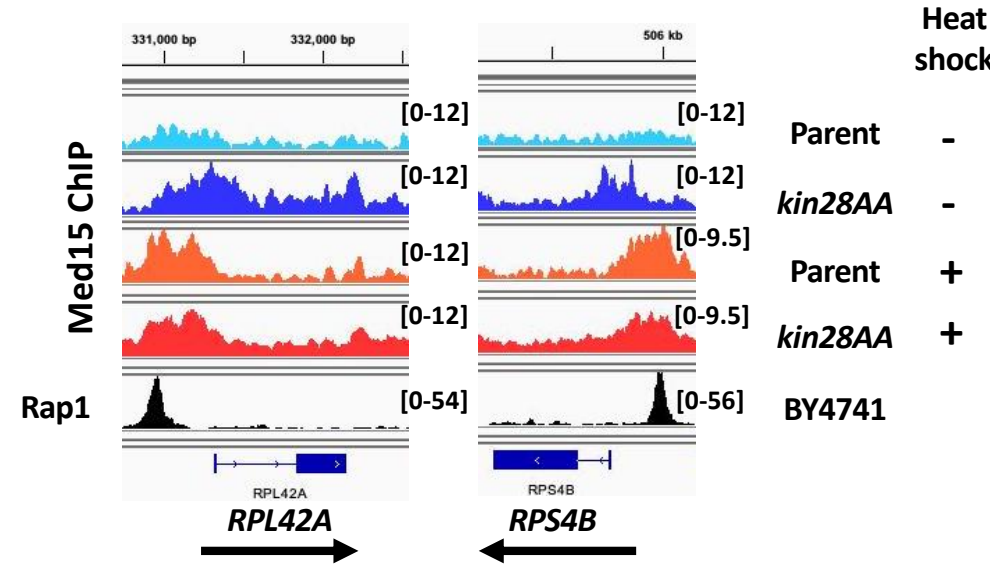

B

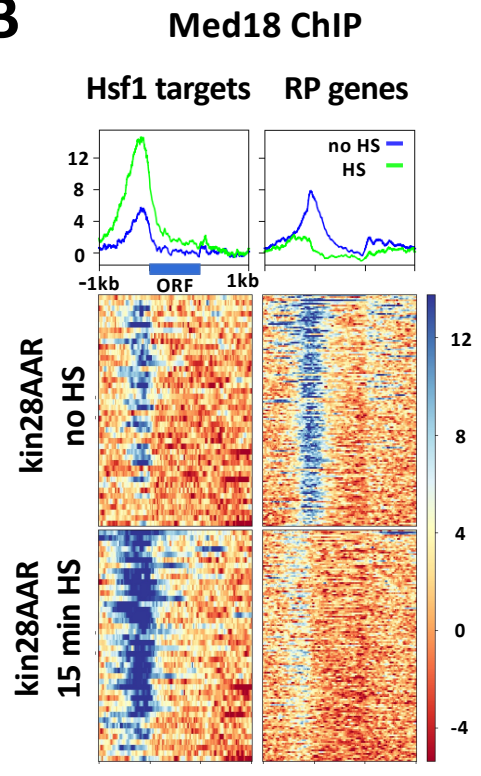

C BY4741

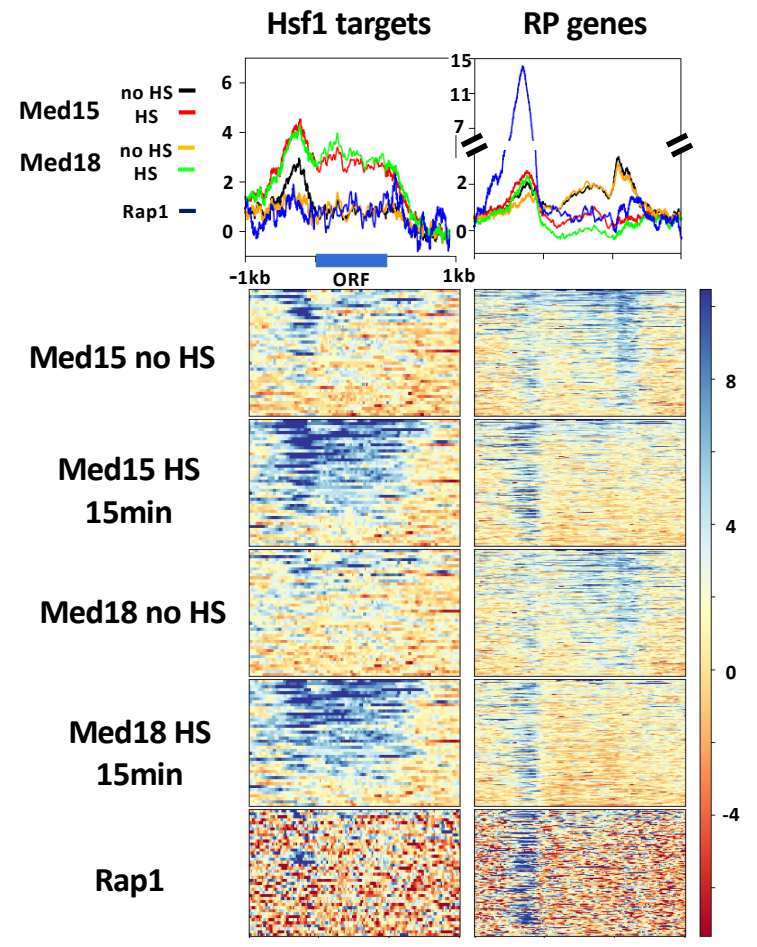


Figure 4

A

YFR1321

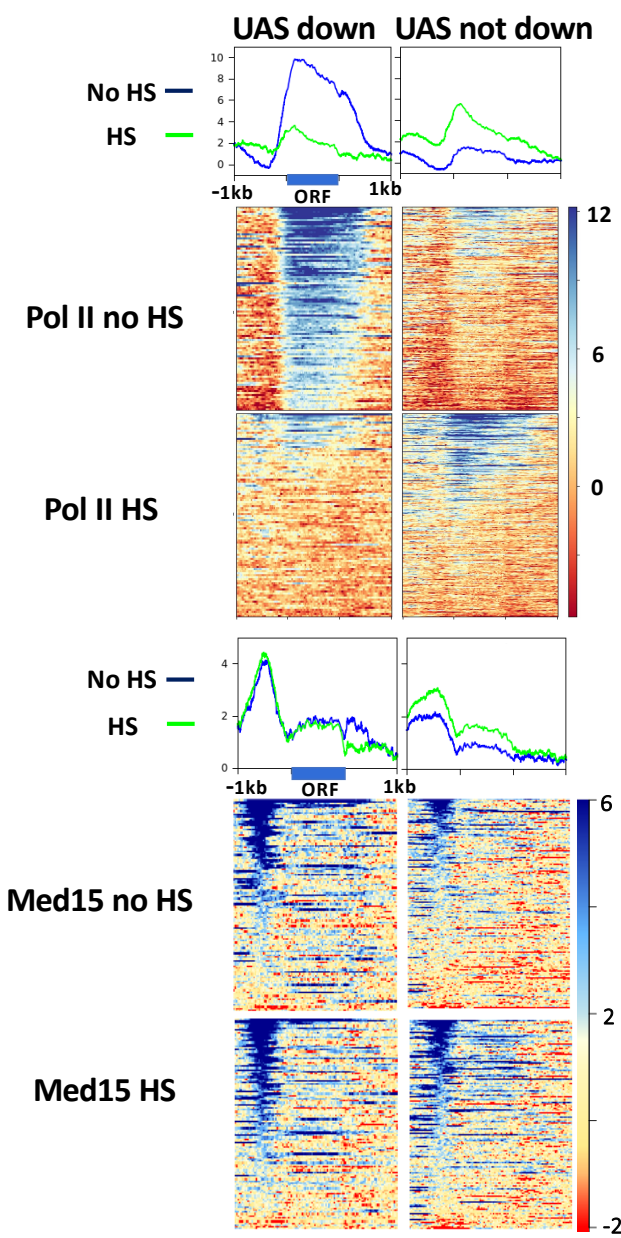

B

$\operatorname{kin} 28-A A$

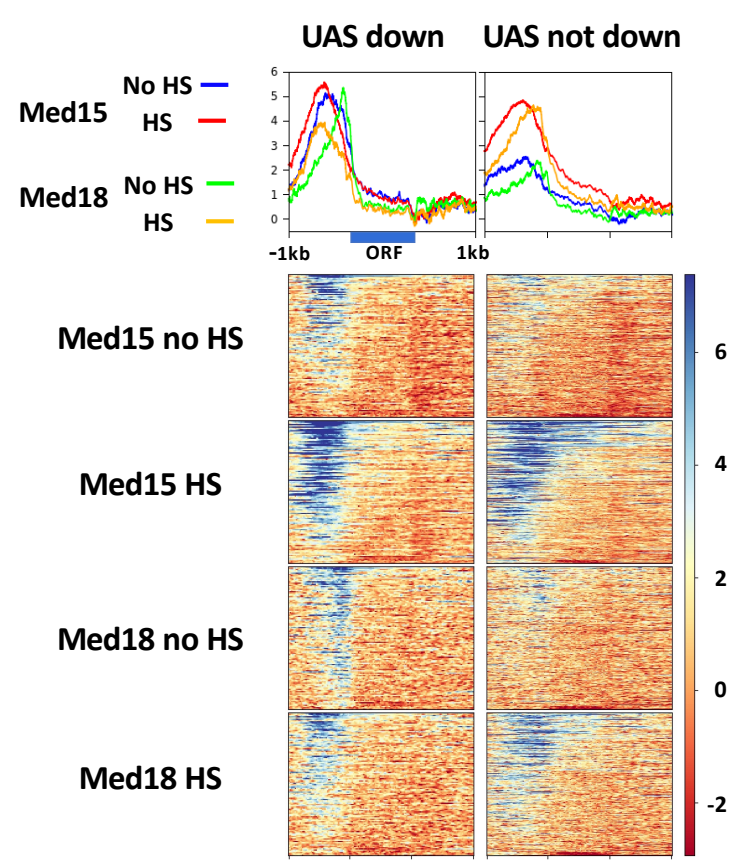

C

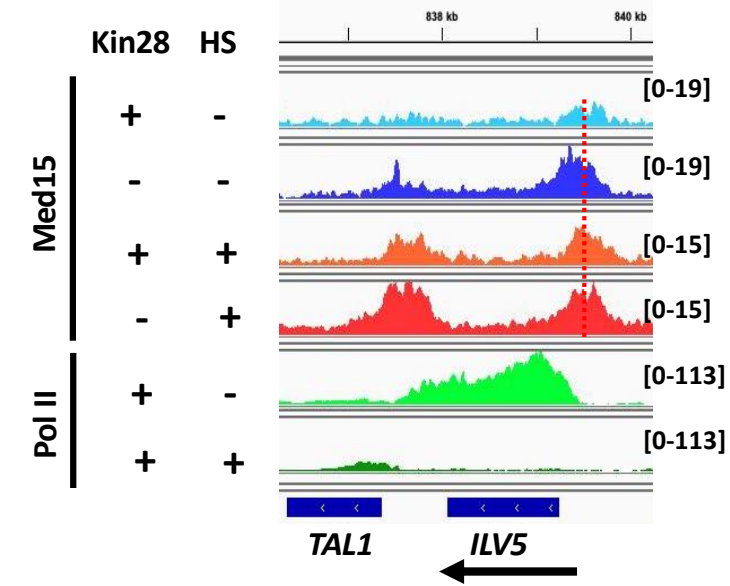

Kin28 HS

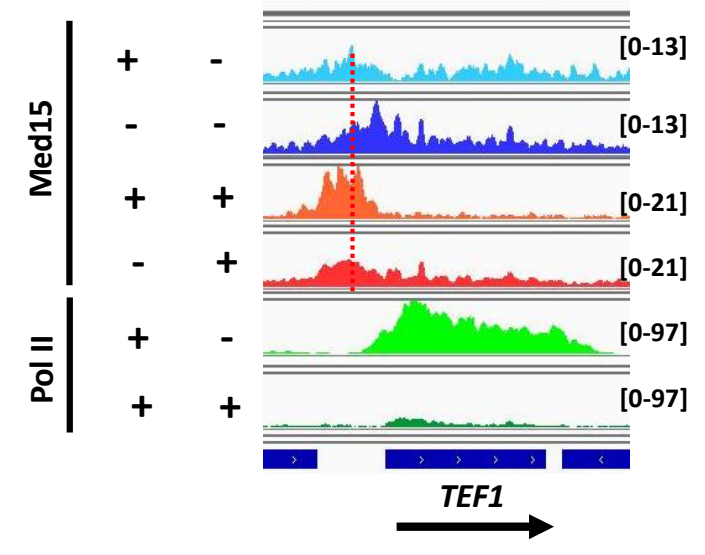


bioRxiv preprint doi: https://doi.org/10.1101/2020.08.25.267088; this version posted August 26, 2020. The copyright holder for this preprint (which was not certified by peer review) is the author/funder. This article is a US Government work. It is not subject to copyright under 17 USC 105 and is also made available for use under a CCO license.

\section{Figure 5}

A

\section{Med15 ChIP}

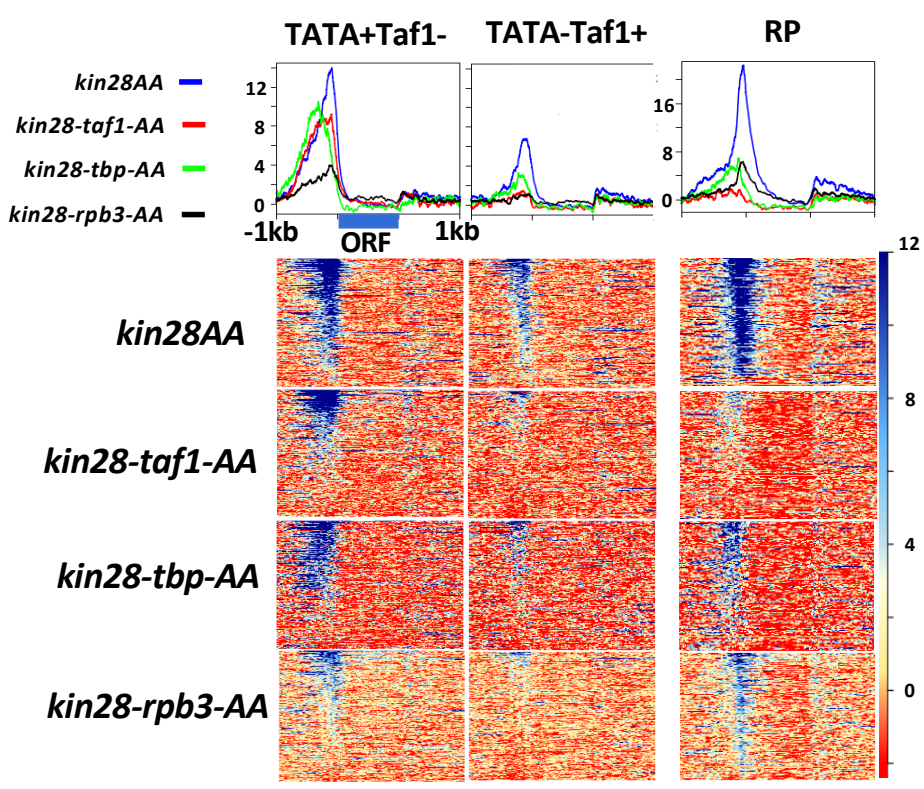

Med18 ChIP

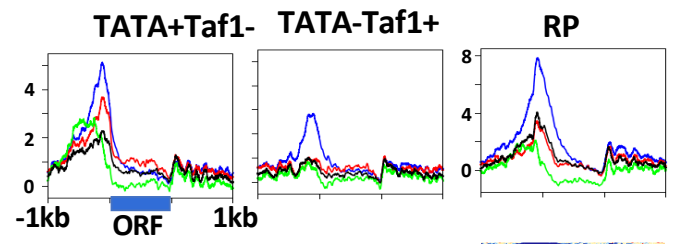

B

\section{Med15 ChIP}

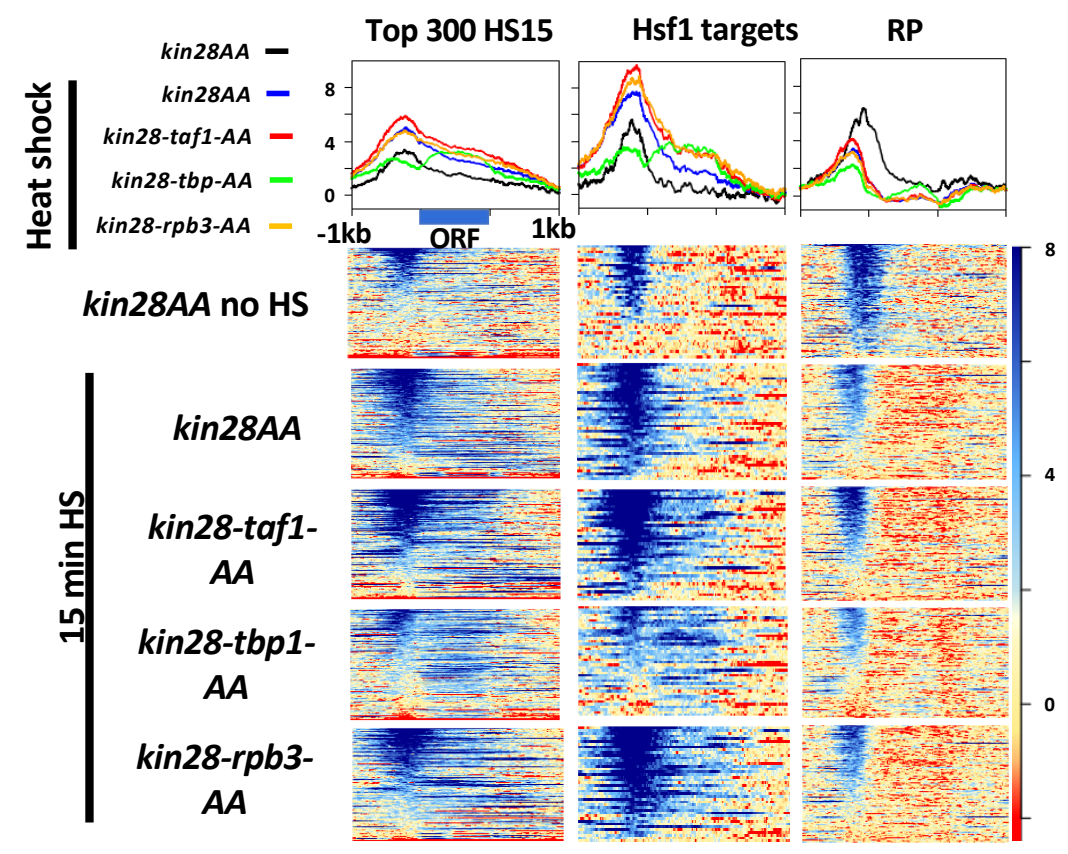

\section{Med18 ChIP}

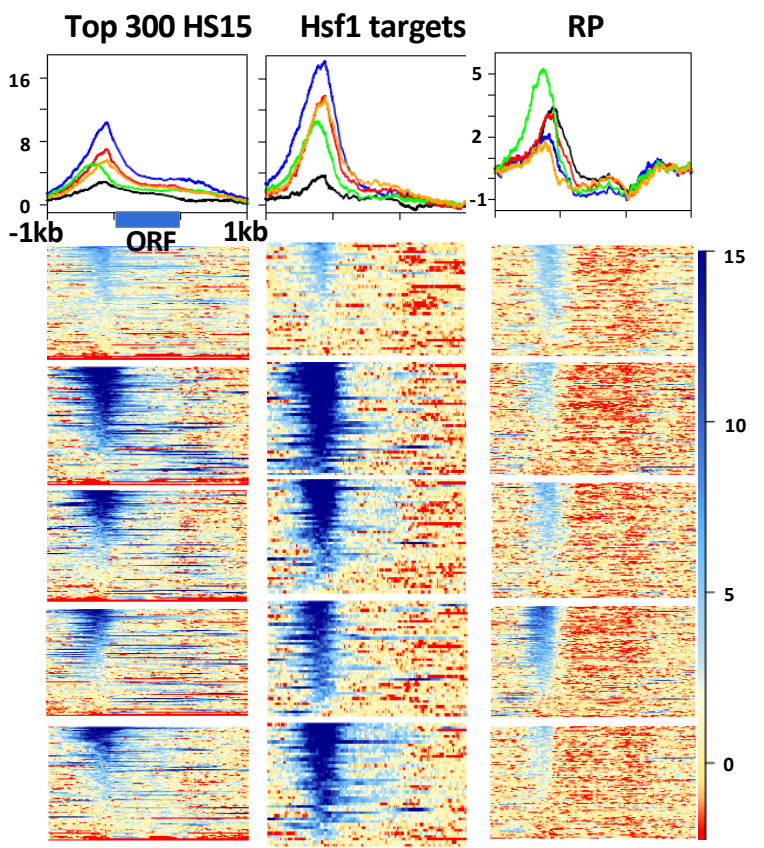




\section{Figure 6}
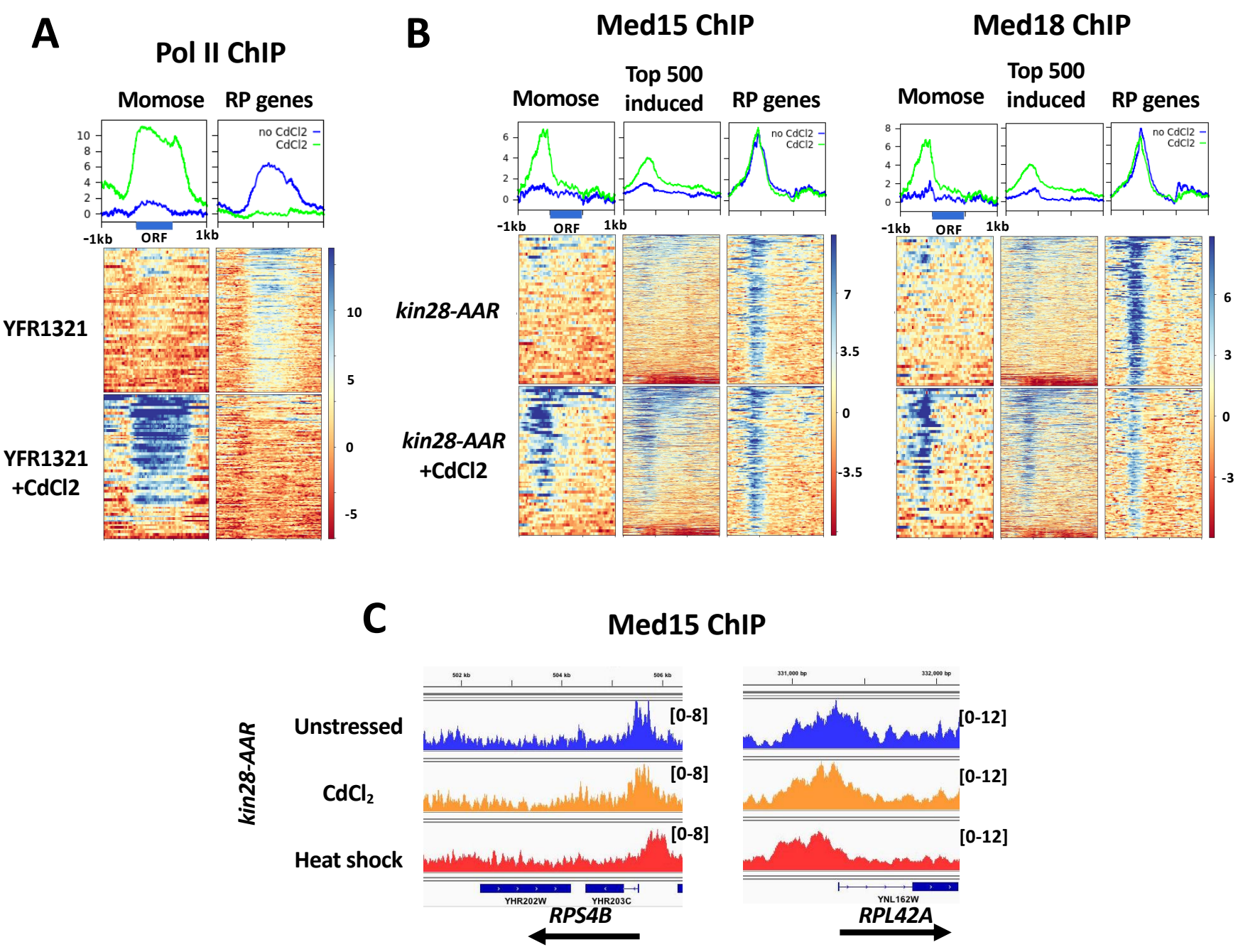


\section{Figure 7}

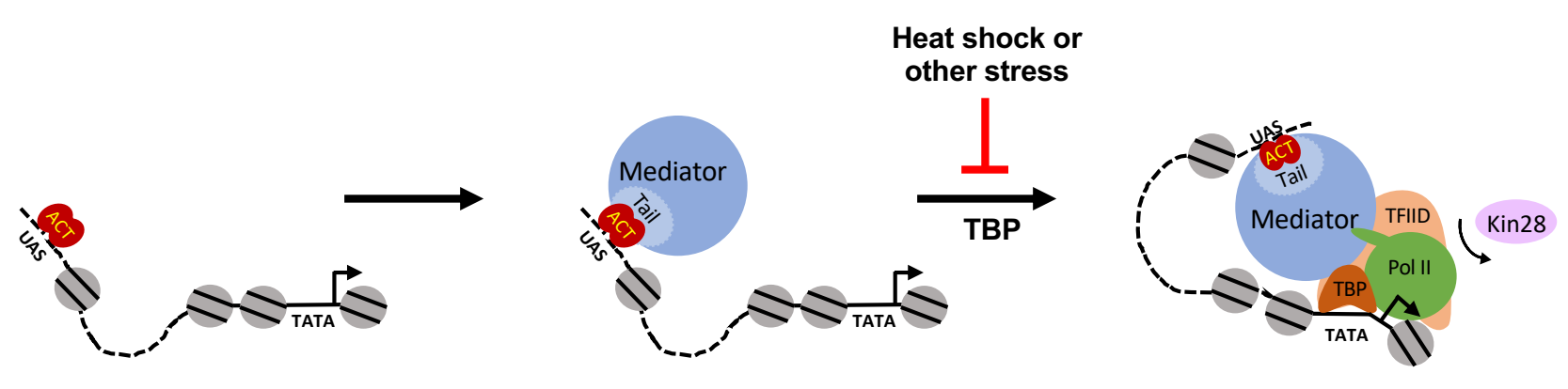

